\title{
HOMOGENEITY AND PRIME MODELS IN TORSION-FREE HYPERBOLIC GROUPS
}

\author{
A. OULD HOUCINE \\ Université de Mons, Institut de Mathématique, \\ Bâtiment Le Pentagone, \\ Avenue du Champ de Mars 6, \\ B-7000 Mons, Belgique \\ Université de Lyon, INSA de Lyon, \\ F-69621, Ecole Centrale de Lyon, CNRS, \\ UMR5208, Institut Camille Jordan, \\ 43 Blvd du 11 Novembre 1918, \\ F-69622 Villeurbanne-Cedex, France \\ ould@math.univ-lyon1.fr \\ Received 2 December 2010 \\ Revised 4 March 2011
}

\begin{abstract}
We show that any nonabelian free group $F$ of finite rank is homogeneous; that is for any tuples $\bar{a}, \bar{b} \in F^{n}$, having the same complete $n$-type, there exists an automorphism of $F$ which sends $\bar{a}$ to $\bar{b}$.

We further study existential types and show that for any tuples $\bar{a}, \bar{b} \in F^{n}$, if $\bar{a}$ and $\bar{b}$ have the same existential $n$-type, then either $\bar{a}$ has the same existential type as the power of a primitive element or there exists an existentially closed subgroup $E(\bar{a})$ (respectively $E(\bar{b})$ ) of $F$ containing $\bar{a}$ (respectively $\bar{b}$ ) and an isomorphism $\sigma: E(\bar{a}) \rightarrow E(\bar{b})$ with $\sigma(\bar{a})=\bar{b}$.

We will deal with non-free two-generated torsion-free hyperbolic groups and we show that they are $\exists$-homogeneous and prime. In particular, this gives concrete examples of finitely generated groups which are prime and not quasi axiomatizable, giving an answer to a question of A. Nies.
\end{abstract}

Keywords: Homogeneity; free groups; prime models; torsion-free hyperbolic groups; rigid groups.

\section{Introduction}

From a model-theoretical point of view, homogeneity can be seen as a kind of saturation. For instance, a countable model in a countable language is saturated, if and only if, it is homogeneous and realizes all types of its complete theory. Homogeneity is also a notion related to prime models and it is well known that a countable prime model in a countable language is homogeneous.

It is easy to see that a free group is not saturated. Consequently, it is natural to ask if any free group is at least homogeneous. This question was studied in the case of the free group of rank 2 in [15], where Nies proved that this last group is $\exists$-homogeneous and not prime. 
In this paper, we study the homogeneity of free groups of higher rank and that of particular torsion-free hyperbolic groups including two-generated ones. The study of these last groups is greatly motivated by the previous result of Nies, where the proof seems to use strongly the two-generation property. We emphasize that, as a result of Delzant [4], any (torsion-free) hyperbolic group is embeddable in a twogenerated (torsion-free) hyperbolic group. In some sense, these last groups can have a very complicated structure.

Let $\mathcal{M}$ be a model, $P$ a subset of $\mathcal{M}$ and $\bar{a}$ a tuple from $\mathcal{M}$. The type (respectively existential type) of $\bar{a}$ over $P$, denoted $t p^{\mathcal{M}}(\bar{a} \mid P)$ (respectively $t p_{\exists}^{\mathcal{M}}(\bar{a} \mid P)$ ), is the set of formulas $\varphi(\bar{x})$ (respectively existential formulas $\varphi(\bar{x})$ ) with parameters from $P$ such that $\mathcal{M}$ satisfies $\varphi(\bar{a})$.

A countable model $\mathcal{M}$ is called homogeneous (respectively $\exists$-homogeneous), if for any tuples $\bar{a}, \bar{b}$ of $\mathcal{M}^{n}$, if $t p^{\mathcal{M}}(\bar{a})=t p^{\mathcal{M}}(\bar{b})$ (respectively $t p_{\exists}^{\mathcal{M}}(\bar{a})=t p_{\exists}^{\mathcal{M}}(\bar{b})$ ), then there exists an automorphism of $\mathcal{M}$ which sends $\bar{a}$ to $\bar{b}$. We note, in particular, that $\exists$-homogeneity implies homogeneity. For further notions of homogeneity, we refer the reader to $[8,14]$.

We recall also that a model $\mathcal{M}$ is said to be prime, if it is elementary embeddable in every model of its complete theory. As usual, to axiomatize group theory, we use the language $\mathcal{L}=\left\{.{ }^{-1}, 1\right\}$, where . is interpreted as multiplication, ${ }^{-1}$ is interpreted as the function which sends every element to its inverse and 1 is interpreted as the trivial element. The main results of this paper are as follows.

Theorem 1.1. Let $F$ be a nonabelian free group of finite rank. For any tuples $\bar{a}, \bar{b} \in F^{n}$ and for any subset $P \subseteq F$, if $t p^{F}(\bar{a} \mid P)=t p^{F}(\bar{b} \mid P)$ then there exists an automorphism of $F$ fixing $P$ pointwise and sending $\bar{a}$ to $\bar{b}$.

Let $\mathcal{M}$ be a model and $\mathcal{N}$ a submodel of $\mathcal{M}$. The model $\mathcal{N}$ is said to be existentially closed (abbreviated e.c.) in $\mathcal{M}$, if for any quantifier-free formula $\varphi(\bar{x})$ with parameters from $\mathcal{N}$, if $\mathcal{M} \models \exists \bar{x} \varphi(\bar{x})$, then $\mathcal{N} \models \exists \bar{x} \varphi(\bar{x})$.

Definition 1.2. Let $F$ be a free group and let $\bar{a}=\left(a_{1}, \ldots, a_{m}\right)$ be a tuple from $F$. We say that $\bar{a}$ is a power of a primitive element if there exist integers $p_{1}, \ldots, p_{m}$ and a primitive element $u$ such that $a_{i}=u^{p_{i}}$ for all $i$.

Theorem 1.3. Let $F$ be a nonabelian free group of finite rank. Let $\bar{a}, \bar{b} \in F^{n}$ and $P \subseteq F$ such that $t p_{\exists}^{F}(\bar{a} \mid P)=t p_{\exists}^{F}(\bar{b} \mid P)$. Then either $\bar{a}$ has the same existential type as a power of a primitive element, or there exists an existentially closed subgroup $E(\bar{a})$ (respectively $E(\bar{b})$ ) containing $P$ and $\bar{a}$ (respectively $\bar{b}$ ) and an isomorphism $\sigma: E(\bar{a}) \rightarrow E(\bar{b})$ fixing $P$ pointwise and sending $\bar{a}$ to $\bar{b}$.

A group $\Gamma$ is said to be co-hopfian, if any injective endomorphism of $\Gamma$ is an automorphism. In [35], Sela proved that a non-cyclic freely indecomposable torsion-free 
hyperbolic group is co-hopfian. In fact, when the given group is two-generated, we have a stronger property. We introduce the following definition.

Definition 1.4. A group $\Gamma$ is said to be strongly co-hopfian, if there exists a finite subset $S \subseteq \Gamma \backslash\{1\}$ such that for any endomorphism $\varphi$ of $\Gamma$, if $1 \notin \varphi(S)$ then $\varphi$ is an automorphism.

For instance, Tarski monster groups are strongly co-hopfian. Recall that a Tarski monster group is an infinite group in which every nontrivial proper subgroup is cyclic of order $p$, where $p$ is a fixed prime. Such groups were built by Ol'shanskiu in [24] and for more details we refer the reader to [25]. It is easily seen that they are simple. It is an immediate consequence that a nontrivial endomorphism of a Tarski Monster group is an automorphism and thus such a group is strongly co-hopfian.

Theorem 1.5. A non-free two-generated torsion-free hyperbolic group is strongly co-hopfian.

The proof of Theorem 1.5 is related to the properties of $\Gamma$-limit groups and to the special properties of two-generated hyperbolic groups. We will also use the following notion.

Definition 1.6. ([18, Definition 3.4]) A $\Gamma$-limit group $G$ is said to be $\Gamma$-determined if there exists a finite subset $S \subseteq G \backslash\{1\}$ such that for any homomorphism $f: G \rightarrow L$, where $L$ is a $\Gamma$-limit group, if $1 \notin f(S)$ then $f$ is an embedding.

From Theorem 1.5, we deduce the following.

Corollary 1.7. A non-free two-generated torison-free hyperbolic group $\Gamma$ is $\exists$ homogeneous, prime and $\Gamma$-determined.

The above enables one to give examples of one-relator $\exists$-homogeneous and prime groups. Indeed, in the free group $F=\langle a, b \mid\rangle$ if we let $r \in F$ such that $r$ is root-free and the symmetrized set that it generates satisfies the small cancellation condition $C^{\prime}(1 / 6)$, then the group $\Gamma=\langle a, b \mid r=1\rangle$ is a non-free two-generated torsion-free one-relator hyperbolic group, which is consequently $\exists$-homogeneous and prime.

Rigid groups are defined in [33] and an equivalent definition in our context is that a torsion-free hyperbolic group $\Gamma$ is rigid if it is freely indecomposable and does not admit an essential cyclic splitting. Following [7, Definition 7.1], a finitely generated subgroup $H \leq \Gamma$ is called immutable if there are finitely many embeddings $\varphi_{1}, \ldots, \varphi_{n}: H \rightarrow \Gamma$ so that any embedding $\varphi: H \rightarrow \Gamma$ is conjugate to one of the $\varphi_{i}$. It follows from [7, Lemma 7.2] that a subgroup $H \leq \Gamma$ is immutable if and only if it is rigid. We note that a torsion-free hyperbolic group is an immutable subgroup of itself if and only if it is rigid. We point out that a rigid torsion-free hyperbolic group is strongly co-hopfian and in particular $\exists$-homogeneous and prime, as well as co-hopfian immutable subgroups of torsion-free hyperbolic groups (see Lemmas 3.3 and 3.5$)$. 
As mentioned in [7] (see [1]), the fundamental group of a closed hyperbolic $n$-manifold where $n \geq 3$ is rigid and thus it is $\exists$-homogeneous and prime. Hence this gives examples of $\exists$-homogeneous torsion-free hyperbolic groups which are not necessarily two-generated.

We notice that Corollary 1.7 shows also that the Cantor-Bendixson rank of a two-generated torsion-free hyperbolic group is 0 in the space of its limit groups. For more details on this notion, we refer the reader to $[21,20]$.

Following [16], a finitely generated group $\Gamma$ is said to be QFA (quasi-finitely axiomatizable) if there exists a sentence $\varphi$ satisfied by $\Gamma$ such that any finitely generated group satisfying $\varphi$ is isomorphic to $\Gamma$. Nies [16] has proved that the free nilpotent group of class 2 with two generators is QFA and prime. Oger and Sabbagh show that finitely generated nonabelian free nilpotent groups are QFA and prime [26]. In [17], the existence of continuously many non-isomorphic finitely generated prime groups is proved, which implies that there exists a finitely generated group which is prime but not QFA. However, the groups built by Nies are quasi axiomatizable. Let us remind that a finitely generated group $G$ is said to be quasi axiomatizable if any finitely generated group which is elementary equivalent to $G$ is isomorphic to $G$ [17]. Consequently, Nies raised the problem of the existence of a prime group which is not quasi axiomatizable [17].

Corollary 1.7 gives concrete examples of finitely generated groups which are prime and not quasi axiomatizable. Indeed, it follows from [39], that if $\Gamma$ is a nonfree two-generated torsion-free hyperbolic group, then $\Gamma$ is an elementary subgroup of $\Gamma * \mathbb{Z}$. By using Grushko theorem $\Gamma$ is not isomorphic to $\Gamma * \mathbb{Z}$, and thus $\Gamma$ is not quasi axiomatizable (see Sec. 8, Proposition 8.7).

The present paper is organized as follows. In Sec. 2, we record the material that we require around $\Gamma$-limit groups and the tools needed in the sequel. In Sec. 3, we present preliminary propositions. Section 4 concerns existential types and the proof of Theorem 1.3 when $P=\emptyset$. Section 5 is devoted to the general case and we show Theorem 1.1 when $P=\emptyset$. Section 6 deals with parameters and the proof of Theorems 1.1 and 1.3 for any $P$. Section 7 is devoted to the proof of Theorem 1.5 and Corollary 1.7. Section 8 concludes with some remarks and we show, in particular, that non-cyclic torsion-free hyperbolic groups are connected.

Remark 1.8. When the work presented in this paper was under verification and more thorough investigation, preprint [32] appeared in which Perin and Sklinos show the homogeneity of countable free groups and give a counter-example in the case of torsion-free hyperbolic groups. The method which we use in this paper is different from that used in [32].

\section{Prerequisites}

We recall some material about $\Gamma$-limit groups, where $\Gamma$ is a torsion-free hyperbolic group, developed by Sela [39]. For more details, we refer the reader to [39]; see also $[7,28]$. We begin by giving some basic definitions. 
Definition 2.1. Let $\Gamma$ be a group and $H$ a finitely generated group.

(1) A sequence of homomorphisms $\left(f_{n}\right)_{n \in \mathbb{N}}$ from $H$ to $\Gamma$ is called stable if, for any $h \in H$, either $f_{n}(h)=1$ for all but finitely many $n$, or $f_{n}(h) \neq 1$ for all but finitely many $n$. The stable kernel of $\left(f_{n}\right)_{n \in \mathbb{N}}$, denoted $\operatorname{Ker}_{\infty}\left(f_{n}\right)$, is the set of elements $h \in H$ such that $f_{n}(h)=1$ for all but finitely many $n$.

(2) A $\Gamma$-limit group is a group $G$ such that there exists a finitely generated group $H$ and a stable sequence of homomorphisms $\left(f_{n}\right)_{n \in \mathbb{N}}$ from $H$ to $\Gamma$ such that $G=H / \operatorname{Ker}_{\infty}\left(f_{n}\right)$.

The following lemma explains the relation between the previous notion, which comes essentially from geometrical considerations, and the universal theory of the considered group. For the definition of universal theories, we refer the reader to $[8,14]$ or $[18]$.

Lemma 2.2. Let $\Gamma$ be a group. A finitely generated group is $\Gamma$-limit if and only if it is a model of the universal theory of $\Gamma$.

Proof. Let $G$ be a group, generated by a finite tuple $\bar{a}$. Suppose that $G$ is $\Gamma$-limit and let us show that $G$ satisfies the universal theory of $\Gamma$. To this end, it is sufficient to show that every existential sentence true in $G$ is true in $\Gamma$.

Let

$$
\varphi:=\exists \bar{x} \bigvee_{1 \leq i \leq n}\left(\bigwedge_{w \in P_{i}} w(\bar{x})=1 \wedge \bigwedge_{v \in N_{i}} v(\bar{x}) \neq 1\right),
$$

where $P_{i}$ and $N_{i}$ are finite sets of words, be an existential sentence satisfied by $G$. Then there exists a tuple of words $\bar{p}(\bar{y})$ and $1 \leq q \leq n$, such that

$$
G \models \bigwedge_{w \in P_{q}} w(\bar{p}(\bar{a}))=1 \wedge \bigwedge_{v \in N_{q}} v(\bar{p}(\bar{a})) \neq 1 .
$$

Let $H$ be a finitely generated group and $\left(f_{n}: G \rightarrow \Gamma\right)$ be a stable sequence such that $G=H / \operatorname{Ker}_{\infty}\left(f_{n}\right)$. Let $\pi: H \rightarrow G$ be the natural map and let $\bar{b}$ be a preimage of $\bar{a}$. Since the previous sequence is stable, by (2.1), we conclude that

$$
\Gamma \models \bigwedge_{w \in P_{q}} w\left(\bar{p}\left(f_{n}(\bar{b})\right)\right)=1 \wedge \bigwedge_{v \in N_{q}} v\left(\bar{p}\left(f_{n}(\bar{b})\right)\right) \neq 1,
$$

for all but finitely many $n$ and thus $\Gamma \models \varphi$ as required.

Suppose now that $G$ is a model of the universal theory of $\Gamma$ and let us show that $G$ is $\Gamma$-limit. Let $\left(w_{i}(\bar{x}) \mid i \in \mathbb{N}\right)$ be the sequence of all words such that $G \models w_{i}(\bar{a})=$ 1 , and let $\left(v_{i}(\bar{x}) \mid i \in \mathbb{N}\right)$ be the sequence of all words such that $G \models v_{i}(\bar{a}) \neq 1$.

Since $G$ is a model of the universal theory of $\Gamma$, for any $n \geq 0$, we have

$$
\Gamma \models \exists \bar{x}\left(\bigwedge_{0 \leq i \leq n} w_{i}(\bar{x})=1 \wedge \bigwedge_{0 \leq i \leq n} v_{i}(\bar{x}) \neq 1\right) .
$$


Let $F$ be the free group with basis $\bar{x}$. Hence, for any $n \geq 0$, there exists a homomorphism $f_{n}: F \rightarrow \Gamma$ such that $f_{n}\left(w_{i}(\bar{x})\right)=1$ and $f_{n}\left(v_{i}(\bar{x})\right) \neq 1$ for $0 \leq i \leq n$. We claim that the sequence $\left(f_{n}: F \rightarrow \Gamma\right)$ is stable. Indeed, let $w(\bar{x})$ be a reduced word in $F$. Then either $w=w_{p}$ or $w=v_{p}$ for some $p$. If the first case holds, then $f_{n}(w)=1$ for all $n \geq p$ and if the second case holds then $f_{n}(w) \neq 1$ for all $n \geq p$. Hence the sequence is stable as claimed. Using the same argument, we see that $F / \operatorname{Ker}_{\infty}\left(f_{n}\right)=G$.

Let $G$ be a group and $A$ a subgroup of $G$. The group $G$ is said to be freely $A$-decomposable or freely decomposable relative to $A$, if $G$ has a nontrivial free decomposition $G=G_{1} * G_{2}$ such that $A \leq G_{1}$. Otherwise, $G$ is said to be freely $A$-indecomposable or freely indecomposable relative to $A$.

A cyclic splitting of a group $G$ is a graph of groups decomposition of $G$ in which every edge group is infinite cyclic. A cyclic splitting is said to be essential if any edge group has infinite index in the adjacent vertex groups.

Theorem 2.3. ([39], see also [7, Theorem 3.9]) Let $\Gamma$ be a torsion-free hyperbolic group. Let $H$ be a freely indecomposable finitely generated group and let $\left(f_{n}: H \rightarrow\right.$ $\Gamma)_{n \in \mathbb{N}}$ be a stable sequence of pairwise nonconjugate homomorphisms with trivial stable kernel. Then $H$ admits an essential cyclic splitting.

Sela [38] and Kharlampovich and Myasnikov [11] show that nonabelian free groups have the same elementary theory and, in fact, the following more explicit description.

Theorem 2.4. A nonabelian free factor of a free group of finite rank is an elementary subgroup.

In fact, in [36, 37] and [11] the following quantifier-elimination result is proved.

Theorem 2.5. Let $\varphi(\bar{x})$ be a formula. Then there exists a boolean combination of $\exists \forall$-formulas $\phi(\bar{x})$, such that for any nonabelian free group $F$ of finite rank, one has $F \models \forall \bar{x}(\varphi(\bar{x}) \Leftrightarrow \phi(\bar{x}))$.

We notice, in particular, that if $\bar{a}, \bar{b} \in F^{n}$ such that $t p_{\exists \forall}^{F}(\bar{a})=t p_{\exists \forall}^{F}(\bar{b})$, then $t p^{F}(\bar{a})=t p^{F}(\bar{b})$.

In [28], the converse of Theorem 2.4 is proved.

Theorem 2.6. An elementary subgroup of a free group of finite rank is a free factor.

In [31], Pillay shows the following.

Theorem 2.7. Let $F$ be a nonabelian free group of finite rank and let $u, v \in F$ such that $t p^{F}(u)=t p^{F}(v)$. If $u$ is primitive, then $v$ is primitive. 
In the proof of Theorems 1.1 and 1.3, we use the following properties of free groups. Let $F$ be a free group with a finite basis $A$. Let $|u|$ denote the length of a word $u$ in $F$, with respect to the basis $A$. From [13, Proposition 2.5, Chap. I], a subgroup $H \leq F$ has a Nielsen-reduced generating set $U$ and a Nielsen-reduced set $U$ satisfying the following property [13, Proposition 2.13, Chap. I]: if $w \in H$ has the form $w=u_{1} u_{2} \cdots u_{m}$ where each $u_{i} \in U^{ \pm 1}$ and $u_{i} u_{i+1} \neq 1$ then $|w| \geq m$ and $|w| \geq\left|u_{i}\right|$ for any $i$. Hence we can conclude the previous remarks with the following proposition.

Proposition 2.8. Let $F$ be a nonabelian free group. Then any subgroup $H$ of rank $m$ of $F$ has a basis $B=\left\{b_{1}, \ldots, b_{m}\right\}$ such that for any reduced nontrivial word $w$ on $A$ one has $|w| \geq|b|$ for any $b \in B$ which appears in the reduced form of $w$ with respect to $B$.

Proposition 2.9. ([13, Proposition 2.12]) Let $f$ be a homomorphism from a free group $F$ of finite rank onto a free group $G$. Then $F$ admits a free decomposition $F=A * B$ such that $f(A)=G$ and $f(B)=1$ and $f$ is injective on $A$.

The next proposition is a particular case of [27, Proposition 1].

Proposition 2.10. Let $F$ be a nonabelian free group of finite rank and let $H$ be a nontrivial subgroup of $F$. If $f: F \rightarrow F$ is a non-surjective monomorphism such that $f(H)=H$, then $F$ is freely $H$-decomposable.

In dealing with prime models, the following characterization is useful.

Proposition 2.11. ([8]) Let $\mathcal{M}$ be a countable model. Then $\mathcal{M}$ is a prime model of its theory if and only if for every $m \in \mathbb{N}$, each orbit under the action of $\operatorname{Aut}(\mathcal{M})$ on $\mathcal{M}^{m}$ is first-order definable without parameters.

Recall that a group is said to be equationally noetherian if any system of equations in finitely many variables is equivalent to a finite subsystem. For more details on this notion, we refer the reader to [2]. A theorem of Sela [39, Theorem 1.22] states that any system of equations, without parameters, in finitely many variables is equivalent in a trosion-free hyperbolic group to a finite subsystem. The previous property is equivalent, when the group under consideration $G$ is finitely generated, to the fact that $G$ is equationally noetherian. Indeed, let $G$ be a group, generated by a finite tuple $\bar{a}$, and suppose that any system of equations, without parameters, in finitely many variables is equivalent to a finite subsystem. Let $\left(w_{i}\left(\bar{p}_{i}, \bar{x}\right)=1 \mid i \in I\right)$ be a system of equations with finitely variables $\bar{x}$ and parameters $\bar{p}_{i}$. Since $G$ is finitely generated, for each $i$, there is a tuple of words $\bar{p}_{i}(\bar{y})$ such that $\bar{p}_{i}=\bar{p}_{i}(\bar{a})$. Now, the new system $\left(w_{i}\left(\bar{p}_{i}(\bar{y}), \bar{x}\right)=1 \mid i \in I\right)$ is without parameters and thus it is equivalent to a finite subsystem $\left(w_{i}\left(\bar{p}_{i}(\bar{y}), \bar{x}\right)=1 \mid i \in I_{0}\right)$, where $I_{0}$ is finite. Therefore $\left(w_{i}\left(\bar{p}_{i}(\bar{a}), \bar{x}\right)=1 \mid i \in I\right)$ is equivalent to $\left(w_{i}\left(\bar{p}_{i}(\bar{a}), \bar{x}\right)=1 \mid i \in I_{0}\right)$.

Hence, we have the following.

Theorem 2.12. A torsion-free hyperbolic group is equationally noetherian. 


\section{Preliminaries}

Recall that a subgroup $A$ of a group $G$ is said to be malnormal if for any $g \in G \backslash A$, $A^{g} \cap A=1$. A group $G$ is said to be a $C S A$-group, if any maximal abelian subgroup of $G$ is malnormal. In particular, a CSA-group is commutative transitive; that is, the commutation is a transitive relation on the set of nontrivial elements. Basic facts about CSA-groups and their HNN-extensions will be used freely throughout the rest of the paper. For more details, see [19, 9, 22]. In an HNN-extension we denote by $|g|$ the length of normal forms of $g$.

Lemma 3.1. Let $G=\left\langle H, t \mid U^{t}=V\right\rangle$ where $U$ and $V$ are cyclic subgroups of $G$ generated respectively by $u$ and $v$. Suppose that:

(i) $U$ and $V$ are malnormal in $H$.

(ii) $U^{h} \cap V=1$ for any $h \in H$.

Let $\alpha, \beta \in H, s \in G$ such that $\alpha^{s}=\beta,|s| \geq 1$. Then one of the following cases holds:

(1) $\alpha=u^{p \gamma}, \beta=v^{p \delta}, s=\gamma^{-1} t \delta$, where $p \in \mathbb{Z}$ and $\gamma, \delta \in H$.

(2) $\alpha=v^{p \gamma}, \beta=u^{p \delta}, s=\gamma^{-1} t^{-1} \delta$, where $p \in \mathbb{Z}$ and $\gamma, \delta \in H$.

Proof. Write $s=h_{0} t^{\epsilon_{0}} \cdots t^{\epsilon_{n}} h_{n+1}$ in normal form. Hence

$$
h_{n+1}^{-1} t^{-\epsilon_{n}} \cdots t^{-\epsilon_{0}} h_{0}^{-1} \alpha h_{0} t^{\epsilon_{0}} \cdots t^{\epsilon_{n}} h_{n+1}=\beta,
$$

and thus either $h_{0}^{-1} \alpha h_{0} \in U$ and $\epsilon_{0}=1$ or $h_{0}^{-1} \alpha h_{0} \in V$ and $\epsilon_{0}=-1$.

We treat only the first case, the other case can be treated similarly. Therefore $\alpha=h_{0} u^{p} h_{0}^{-1}$ for some $p \in \mathbb{Z}$.

We claim that $n=0$. Suppose that $n \geq 1$. Then $h_{1}^{-1} v^{p} h_{1} \in U$ and $\epsilon_{1}=1$ or $h_{1}^{-1} v^{p} h_{1} \in V$ and $\epsilon_{1}=-1$. Since $U^{h} \cap V=1$, the first case is impossible. Therefore we have the second case and thus $h_{1} \in V$ by the malnormality of $V$. Hence the sequence $\left(t^{\epsilon_{0}}, h_{1}, t^{\epsilon_{1}}\right)$ is not reduced, which is a contradiction. Thus $n=0$ as claimed and hence $\alpha=h_{0} u^{p} h_{0}^{-1}, s=h_{0} t h_{1}, \beta=h_{1}^{-1} v^{p} h_{1}$.

In [12] the structure of two-generated torsion-free hyperbolic groups was investigated. The next theorem is a version of [12, Proposition 5.3]. The proof is essentially the same. We remove the occurrence of free products with amalgamation and we show that the cyclic subgroups involved can be chosen to be malnormal in the vertex group.

Theorem 3.2. Let $\Gamma$ be a non-free two-generated torsion-free hyperbolic group. Then there exists a sequence of subgroups $\Gamma=\Gamma_{1} \geq \Gamma_{2} \geq \cdots \geq \Gamma_{n}$ satisfying the following properties:

(i) Each $\Gamma_{i}$ is two-generated, hyperbolic and quasi-convex;

(ii) $\Gamma_{i}=\left\langle\Gamma_{i+1}, t \mid A^{t}=B\right\rangle$, where $A$ and $B$ are nontrivial malnormal cyclic subgroups of $\Gamma_{i+1}$;

(iii) $\Gamma_{n}$ is a rigid subgroup of $\Gamma$. 
Proof. The construction of the sequence proceeds as follows. If $\Gamma$ is rigid, then we get our sequence by setting $n=1$. So we assume that $\Gamma$ is not rigid. Hence $G$ admits an essential cyclic splitting. By [3, Proposition 4.26], $\Gamma$ admits an abelian splitting in which each edge group is maximal in the neighboring vertex group. Since $\Gamma$ is a torsion-free hyperbolic group, any abelian subgroup is cyclic and thus $\Gamma$ admits a cyclic splitting in which each edge group is malnormal in the neighboring vertex group. Since that splitting is cyclic and essential, it has only one vertex and one edge by $[12$, Theorem $\mathrm{A}]$. Hence, $\Gamma$ admits a splitting of the form

$$
\Gamma=\left\langle K, t \mid A^{t}=B\right\rangle
$$

where $A$ and $B$ are malnormal nontrivial cyclic subgroups of $K$. By [12, Proposition $3.8], K$ is two-generated and freely indecomposable. It is also quasi-convex by [12, Proposition 4.5] and hence hyperbolic. We set $\Gamma_{2}=K$.

Now $\Gamma_{2}$ satisfies the same properties as $\Gamma$ and we can apply the same procedure to it as above. As in the proof of [12, Proposition 5.3], we show that there exists $p \in \mathbb{N}$ so that for any sequence of subgroups $\Gamma_{1}=\Gamma \geq \Gamma_{2} \geq \cdots \geq \Gamma_{n}$ satisfying (i) and (ii) of the theorem we have $n \leq p$. By a result of Delzant [4], the number of distinct conjugacy classes of two-generated freely indecomposable subgroups in a torsion-free hyperbolic group is finite. Let $p$ be that number. Suppose that $n>p$. Then there exists $i<j<n$ such that $\Gamma_{j}=\Gamma_{i}^{g}$ for some $g \in \Gamma$. Therefore $\Gamma_{i}^{g}$ is a proper subgroup of $\Gamma_{i}$; a contradiction with [12, Lemma 4.6] as $\Gamma_{i}$ is quasi-convex subgroup of $\Gamma$.

Hence in a maximal sequence $\Gamma=\Gamma_{1} \geq \Gamma_{2} \geq \cdots \geq \Gamma_{n}$ satisfying (i) and (ii) of the theorem, $\Gamma_{n}$ does not admit an essential splitting and thus is rigid.

Since rigid torsion-free hyperbolic groups are freely indecomposable, their $\exists$-homogeneity and primeness are consequences of the following lemmas of independent interest.

Lemma 3.3. Let $\Gamma$ be a torsion-free hyperbolic group. A rigid $\Gamma$-limit group is $\Gamma$-determined.

Proof. Let $H$ be a rigid $\Gamma$-limit group and suppose as a contradiction that $H$ is not $\Gamma$-determined. Therefore for any finite subset $S \subseteq H \backslash\{1\}$, there exists a non-injective homomorphism $\varphi: H \rightarrow L$, where $L$ is a $\Gamma$-limit group, such that $1 \notin \varphi(S)$. Since $L$ is a $\Gamma$-limit group, we may suppose without loss of generality that $\varphi: H \rightarrow \Gamma$. Write $H \backslash\{1\}$ as an increasing sequence of finite subsets $\left(S_{i}\right)_{i \in \mathbb{N}}$. Thus, there exists a sequence of non-injective homomorphisms $\varphi_{i}: H \rightarrow \Gamma$ such that $1 \notin \varphi_{i}\left(S_{i}\right)$. Clearly, such a sequence is stable and has a trivial stable kernel. Since each $\varphi_{i}$ is non-injective, we can extract a stable subsequence of pairwise nonconjugate homomorphisms with trivial stable kernel. Hence $H$ admits an essential cyclic splitting by Theorem 2.3, which is a contradiction. 
We note that a co-hopfian $\Gamma$-determined group is strongly co-hopfian. We introduce the following definition, which is a light generalization of Definition 1.4.

Definition 3.4. Let $G$ be a group and let $\bar{a}$ be a generating tuple of $G$. We say that $G$ is elementary co-hopfian, if there exists a formula $\varphi(\bar{x})$ such that $G \models \varphi(\bar{a})$ and such that for any endomorphism $h$ of $G$, if $G \models \varphi(h(\bar{a}))$ then $h$ is an automorphism.

We emphasize that the above definition is independent of the chosen generating tuple $\bar{a}$ and that a strongly co-hopfian group is elementary co-hopfian.

Lemma 3.5. Let $\Gamma$ be a finitely generated group which is either equationally noetherian or finitely presented.

(i) If $\Gamma$ is elementary co-hopfian then $\Gamma$ is a prime model.

(ii) If $\Gamma$ is strongly co-hopfian then it is $\exists$-homogeneous, prime and $\Gamma$-determined.

(iii) If $\Gamma$ is equationally noetherian then any co-hopfian $\Gamma$-determined group is $\exists$-homogeneous and prime.

Proof. Let

$$
\Gamma=\left\langle\bar{x} \mid w_{i}(\bar{x})=1, i \in \mathbb{N}\right\rangle
$$

be a presentation of $\Gamma$. Since $\Gamma$ is either equationally noetherian or finitely presented, there exists $p \in \mathbb{N}$ such that

$$
\Gamma \models \forall \bar{x}\left(w_{1}(\bar{x})=1 \wedge \cdots \wedge w_{p}(\bar{x})=1 \Rightarrow w_{i}(\bar{x})=1\right),
$$

for any $i \in \mathbb{N}$.

Proof of (i). Let $\varphi(\bar{x})$ be as in Definition 3.4. Using (1), we conclude that for any tuple $\bar{y}$ in $\Gamma$ which satisfies

$$
w_{1}(\bar{y})=1 \wedge \cdots \wedge w_{p}(\bar{y})=1 \wedge \varphi(\bar{y})
$$

there is an automorphism $f$ of $\Gamma$ which sends $\bar{x}$ to $\bar{y}$.

Let $\bar{b}$ be in $\Gamma^{m}$ and let us show that the orbit of $\bar{b}$ under the action of $A u t(\Gamma)$ is definable. We conclude by Proposition 2.11 .

There exists a tuple of words $\bar{t}(\bar{x})$ such that $\bar{b}=\bar{t}(\bar{x})$. We see that the orbit of $\bar{b}$ is definable by the formula

$$
\psi(\bar{z}):=\exists \bar{y}\left(\bigwedge_{1 \leq i \leq p} w_{i}(\bar{y})=1 \wedge \varphi(\bar{y}) \wedge \bar{z}=\bar{t}(\bar{y})\right) .
$$

Proof of (ii). Since $\Gamma$ is strongly co-hopfian, there exists a finite number of words $v_{1}(\bar{x}), \ldots, v_{m}(\bar{x})$ such that

$$
\Gamma \models v_{i}(\bar{x}) \neq 1,
$$


and such that if $f$ is an endomorphism of $\Gamma$ such that $v_{i}(f(\bar{x})) \neq 1$, for every $1 \leq i \leq m$, then $f$ is an automorphism.

Therefore, using Lemma 2.2, for any $\Gamma$-limit group $L$, if $f: \Gamma \rightarrow L$ is a homomorphism such that $v_{i}(f(\bar{x})) \neq 1$ for every $1 \leq i \leq m$, then $f$ is an embedding. Hence $\Gamma$ is $\Gamma$-determined.

Let us show that $\Gamma$ is $\exists$-homogeneous. We see that for any tuple $\bar{y}$ in $\Gamma$ which satisfies

$$
w_{1}(\bar{y})=1 \wedge \cdots \wedge w_{p}(\bar{y})=1 \wedge v_{1}(\bar{y}) \neq 1 \wedge \cdots \wedge v_{m}(\bar{y}) \neq 1,
$$

there is a homomorphism $f: \Gamma \rightarrow \Gamma$ which sends $\bar{x}$ to $\bar{y}$ and such a homomorphism is necessarily an automorphism.

Let $\bar{a}, \bar{b}$ be tuples of $\Gamma$ such that $t p_{\exists}(\bar{a}) \subseteq t p_{\exists}(\bar{b})$ and let us show that there is an automorphism of $\Gamma$ which sends $\bar{a}$ to $\bar{b}$.

Let $\bar{u}$ be a tuple of words such that $\bar{a}=\bar{u}(\bar{x})$. Since $t p_{\exists}(\bar{a}) \subseteq t p_{\exists}(\bar{b})$, there exists $\bar{y}$ such that

$$
\bar{b}=u(\bar{y}), \quad w_{1}(\bar{y})=1 \wedge \cdots \wedge w_{p}(\bar{y})=1 \wedge v_{1}(\bar{y}) \neq 1 \wedge \cdots \wedge v_{m}(\bar{y}) \neq 1,
$$

and thus there is an automorphism of $\Gamma$ which sends $\bar{a}$ to $\bar{b}$ and thus $\Gamma$ is $\exists$ homogeneous as required. We see that a strongly co-hopfian group is elementary co-hopfian and by (i), we conclude that $\Gamma$ is prime.

Proof of (iii). Let $H$ be a co-hopfian $\Gamma$-determined group. We see that $H$ is strongly co-hopfian. By [18, Corollary 2.10], $H$ is equationally noetherian and we conclude by (ii).

Remark 3.6. We note as a consequence that, if a finitely presented simple group is co-hopfian then it is prime. Indeed, if $\Gamma$ is a finitely presented infinite simple cohopfian group, then by taking, in Definition 1.4, $S$ to be reduced to a one nontrivial element $g$, then any homomorphism $\varphi: \Gamma \rightarrow \Gamma$ with $\varphi(g) \neq 1$ is an automorphism and thus $\Gamma$ is strongly co-hopfian.

We conclude this section with the following lemma of independent interest.

Lemma 3.7. Let $F$ be a nonabelian free group of finite rank and let $\bar{a}, \bar{b}$ be tuples from $F$. Then the existence of an automorphism sending $\bar{a}$ to $\bar{b}$ is equivalent to the existence of a monomorphism sending $\bar{a}$ to $\bar{b}$ and a monomorphism sending $\bar{b}$ to $\bar{a}$.

Proof. Let $f$ and $g$ be monomorphisms such that $f(\bar{a})=\bar{b}$ and $g(\bar{b})=\bar{a}$.

Then $g \circ f$ is a monomorphism which fixes $\bar{a}$. If $g \circ f$ is an automorphism, then $g$ is an automorphism and we are done. If $g \circ f$ is not an automorphism, then $\bar{a}$ is in a proper-free factor of $F$ by Proposition 2.10. A similar argument can be used for $\bar{b}$

So we suppose that $\bar{a}$ and $\bar{b}$ are in proper-free factors. Let $F=F_{1} * A=F_{2} * B$ with $\bar{a} \in F_{1}$ and $\bar{b} \in F_{2}$ and such that $F_{1}$ (respectively $F_{2}$ ) does not have a properfree factor containing $\bar{a}$ (respectively $\bar{b}$ ). 
By applying the Grushko theorem to the subgroup $f\left(F_{1}\right)$ with respect to the decomposition $F=F_{2} * B$ and since $\bar{b} \in f\left(F_{1}\right) \cap F_{2}$, we get $f\left(F_{1}\right)=f\left(F_{1}\right) \cap F_{2} * K$ for some subgroup $K$ of $F$.

We claim that $K=1$. Suppose as a contradiction that $K \neq 1$. Hence, by [13, Theorem 1.8., Chap. IV], $F_{1}$ has a decomposition $P * L$ such that $f(P)=f\left(F_{1}\right) \cap F_{2}$ and $f(L)=K$. Since $K \neq 1$, we get $L \neq 1$. Since $f$ is a monomorphism, we get $\bar{a} \in P$; which is clearly a contradiction with the choice of $F_{1}$. Thus $K=1$ and $f\left(F_{1}\right) \leq F_{2}$.

By a similar argument, we have $g\left(F_{2}\right) \leq F_{1}$. As before, $(g \circ f)_{\mid F_{1}}$ is a monomorphism of $F_{1}$ which fixes $\bar{a}$. If $F_{1}$ is cyclic then $(g \circ f)_{\mid F_{1}}$ is the identity and thus an automorphism of $F_{1}$. If $F_{1}$ is noncyclic, then, since $F_{1}$ is freely indecomposable relative to the subgroup generated by $\bar{a},(g \circ f)_{\mid F_{1}}$ is an automorphism by Proposition 2.10. Hence $g_{\mid F_{2}}$ is surjective. In particular, $F_{1}$ and $F_{2}$ have the same rank. Therefore, $f_{\mid F_{1}}$ can be extended to an automorphism of $F$.

Remark 3.8. By using the same method as that of [15], we note that Lemma 3.7 simplifies the proof of the $\exists$-homogeneity of the free group of rank 2 . Let $\bar{u}$ and $\bar{v}$ be tuples from $F_{2}$ such that $t p_{\exists}(\bar{u})=t p_{\exists}(\bar{v})$. Write $F_{2}=\left\langle x_{1}, x_{2} \mid\right\rangle, \bar{u}=\bar{w}\left(x_{1}, x_{2}\right)$. Since $t p_{\exists}(\bar{u}) \subseteq t p_{\exists}(\bar{v})$, there exists $y_{1}, y_{2}$ such that $\left[y_{1}, y_{2}\right] \neq 1, \bar{v}=\bar{w}\left(y_{1}, y_{2}\right)$. Therefore the map defined by $f\left(x_{i}\right)=y_{i}$ is a monomorphism which sends $\bar{u}$ to $\bar{v}$. Similarly, there exists a monomorphism $g$ which sends $\bar{v}$ to $\bar{u}$. We conclude with Lemma 3.7.

\section{The Existential Case}

We begin this section by examining existential types in free groups. The main purpose is to give the proof of Theorem 1.3 with the hypothesis $P=\emptyset$.

Let $F_{1}$ and $F_{2}$ be nonabelian free groups of finite rank and let $\bar{a}$ (respectively $\bar{b})$ be a tuple from $F_{1}$ (respectively $F_{2}$ ). We denote by $\operatorname{Hom}\left(F_{1}\left|\bar{a}, F_{2}\right| \bar{b}\right)$, the set of homomorphisms $f: F_{1} \rightarrow F_{2}$ such that $f(\bar{a})=\bar{b}$. We denote by $r k(H)$ the rank of of a free group $H$.

If $\bar{a}=\left(a_{1}, \ldots, a_{n}\right)$ is a tuple from $F$, we denote by $|\bar{a}|$ the integer

$$
|\bar{a}|=\max \left\{\left|a_{i}\right| \mid 1 \leq i \leq n\right\},
$$

where $\left|a_{i}\right|$ denotes the length of $a_{i}$ with respect to some fixed basis of the ambiant free group $F$. In the rest of this section, we assume that the tuples which we use are finite and have the same length. For a tuple $\bar{a}$ from $F$, we denote by $t p_{\exists}^{F}(\bar{a})$ its existential type and by $t p_{\forall}^{F}(\bar{a})$ its universal type.

Definition 4.1. Let $F_{1}$ and $F_{2}$ be nonabelian free groups of finite rank and let $\bar{a}$ (respectively $\bar{b}$ ) be a tuple from $F_{1}$ (respectively $F_{2}$ ). We say that $(\bar{a}, \bar{b})$ is existentially rigid, if there is no nontrivial free decomposition $F_{1}=A * B$ such that $A$ contains a tuple $\bar{c}$ with $t p_{\exists}^{F_{1}}(\bar{a}) \subseteq t p_{\exists}^{A}(\bar{c}) \subseteq t p_{\exists}^{F_{2}}(\bar{b})$. 


\section{Remark 4.2.}

(1) Since $A$ is an e.c. subgroup of $F_{1}$, we have $t p_{\exists}^{A}(\bar{c})=t p_{\exists}^{F_{1}}(\bar{c})$.

(2) We note that $(\bar{a}, \bar{a})$ is existentially rigid if and only if $\operatorname{tp}_{\exists}^{F_{1}}(\bar{a}) \cup \operatorname{tp} p_{\forall}^{F_{1}}(\bar{a})$ is not realized in any free group having a smaller rank than the rank of $F_{1}$.

(3) If $F$ is a free group of rank 2 , then for any tuples $\bar{a}, \bar{b},(\bar{a}, \bar{b})$ is existentially rigid.

We begin by showing the following proposition.

Proposition 4.3. Let $F_{1}$ and $F_{2}$ be nonabelian free groups of finite rank and let $\bar{a}$ (respectively $\bar{b}$ ) be a tuple from $F_{1}$ (respectively $\left.F_{2}\right)$. Suppose that $(\bar{a}, \bar{b})$ is existentially rigid. Let $\bar{s}$ be a basis of $F_{1}$. Then there exists a quantifier-free formula $\varphi(\bar{x}, \bar{y})$, such that $F_{1} \models \varphi(\bar{a}, \bar{s})$ and such that for any $f \in \operatorname{Hom}\left(F_{1}\left|\bar{a}, F_{2}\right| \bar{b}\right)$, if $F_{2} \models \varphi(\bar{b}, f(\bar{s}))$ then $f$ is an embedding.

Proof. Let $\left(\psi_{i}(\bar{x}, \bar{y}) \mid i \in \mathbb{N}\right)$ be an enumeration of the quantifier-free type of $(\bar{a}, \bar{s})$ and set

$$
\varphi_{n}(\bar{x}, \bar{y})=\bigwedge_{0 \leq i \leq n} \psi_{i}(\bar{x}, \bar{y})
$$

Suppose as a contradiction that for any $n \in \mathbb{N}$, there exists a non-injective homomorphism $f_{n} \in \operatorname{Hom}\left(F_{1}\left|\bar{a}, F_{2}\right| \bar{b}\right)$ such that $F_{2} \models \varphi_{n}\left(\bar{b}, f_{n}(\bar{s})\right)$.

We emphasize the following property which will be used below implicitly. For any subsequence $\left(f_{n_{k}}\right)_{k \in \mathbb{N}}$ and for any $n \in \mathbb{N}, F_{2} \models \varphi_{n}\left(\bar{b}, f_{n_{k}}(\bar{s})\right)$ for all but finitely many $k$.

Since $f_{n} \in \operatorname{Hom}\left(F_{1}\left|\bar{a}, F_{2}\right| \bar{b}\right), \bar{b} \in f_{n}\left(F_{1}\right)$ and since $f_{n}$ is not injective we get $r k\left(f_{n}\left(F_{1}\right)\right)<r k\left(F_{1}\right)$, for all $n$. Using the pigeon hole principle, we extract a subsequence, that we assume to simplify the notation to be $\left(f_{n}\right)_{n \in \mathbb{N}}$ itself, such that $r k\left(f_{n}\left(F_{1}\right)\right)$ is a fixed natural number $r$ for all $n$.

By Proposition 2.8, each $f_{n}\left(F_{1}\right)$ has a basis $\left\{d_{1 n}, \ldots, d_{p_{n} n}, \ldots, d_{r n}\right\}$ such that $\bar{b}$ is contained in the subgroup generated by $\left\{d_{1 n}, \ldots, d_{p_{n} n}\right\}$ and $\left|d_{i n}\right| \leq|\bar{b}|$ for all $1 \leq i \leq p_{n}$ and for all $n$.

Therefore for any $n \in \mathbb{N}$, the set $\left\{d_{1 n}, \ldots, d_{p_{n} n}\right\}$ is contained in the ball of radius $|\bar{b}|$ of $F_{2}$. Thus, using the pigeon hole principle again, we can find a subsequence, that we assume to simplify the notation to be $\left(f_{n}\right)_{n \in \mathbb{N}}$ itself, such that $p_{n}$ is a fixed integer $p$ and $d_{i n}=d_{i}$ for all $n \in \mathbb{N}$ and $1 \leq i \leq p$.

We conclude that for all $n \in \mathbb{N}$,

$$
f_{n}\left(F_{1}\right)=\left\langle d_{1}, \ldots, d_{p}, d_{(p+1) n}, \ldots, d_{r n}\right\rangle
$$

and $\bar{b}$ is in the subgroup with basis $\left\{d_{1}, \ldots, d_{p}\right\}$.

Set $L=\left\langle d_{1}, \ldots, d_{p}, d_{(p+1) 0}, \ldots, d_{r 0}\right\rangle=f_{0}\left(F_{1}\right)$.

Claim 1. $t p_{\exists}^{F_{1}}(\bar{a}) \subseteq t p_{\exists}^{L}(\bar{b})$. 
Proof. Let $\varphi(\bar{x}, \bar{y})$ be a quantifier-free formula such that $F_{1} \models \exists \bar{y} \varphi(\bar{a}, \bar{y})$. Then there exists a tuple of words $\bar{\alpha}(\bar{t})$ such that $F_{1}=\varphi(\bar{a}, \bar{\alpha}(\bar{s}))$. By construction of the sequence $\left(f_{n}\right)_{n \in \mathbb{N}}, F_{2} \models \varphi\left(\bar{b}, \bar{\alpha}\left(f_{n}(\bar{s})\right)\right)$ for all but finitely many $n$.

Since $f_{n}(\bar{s}), \bar{b}$ are in $f_{n}\left(F_{1}\right)$ we get $f_{n}\left(F_{1}\right) \models \varphi\left(\bar{b}, \bar{\alpha}\left(f_{n}(\bar{s})\right)\right)$ for all but finitely many $n$. Therefore $f_{n}\left(F_{1}\right)=\exists \bar{y} \varphi(\bar{b}, \bar{y})$ for all but finitely many $n$.

The morphism $h: f_{n}\left(F_{1}\right) \rightarrow L$ defined by $h\left(d_{i}\right)=d_{i}$ for $1 \leq i \leq p$ and $h\left(d_{j n}\right)=$ $d_{j 0}$ for $p+1 \leq j \leq r$ is an isomorphism which fixes $\bar{b}$.

Since $h$ is an isomorphism which fixes $\bar{b}$, we conclude that $L \models \exists \bar{y} \varphi(\bar{b}, \bar{y})$. Hence $t p_{\exists}^{F_{1}}(\bar{a}) \subseteq t p_{\exists}^{L}(\bar{b})$ as claimed.

Clearly $t p_{\exists}^{L}(\bar{b}) \subseteq t p_{\exists}^{F_{2}}(\bar{b})$. By Proposition $2.9, F_{1}$ has a free decomposition $F_{1}=$ $A * B$ such that $f_{0}(A)=L$ and $f_{0}(B)=1$ and where $f_{0}$ is injective in restriction to $A$. Since $r k(L)<r k\left(F_{1}\right)$, the preceding decomposition is nontrivial.

Let $\bar{c}$ be the unique tuple of $A$ such that $f_{0}(\bar{c})=\bar{b}$. Since $f_{0}$ is injective in restriction to $A$, we get $t p_{\exists}^{A}(\bar{c})=t p_{\exists}^{L}(\bar{b})$.

We conclude that $t p_{\exists}^{F_{1}}(\bar{a}) \subseteq t p_{\exists}^{A}(\bar{c}) \subseteq t p_{\exists}^{F_{2}}(\bar{b})$ and thus $(\bar{a}, \bar{b})$ is not existentially rigid; which is a final contradiction.

Definition 4.4. Let $F$ be a free group and let $\bar{a}=\left(a_{1}, \ldots, a_{m}\right)$ be a tuple from $F$. We say that $\bar{a}$ is a power of a primitive element, if there exist integers $p_{1}, \ldots, p_{m}$ and a primitive element $u$ such that $a_{i}=u^{p_{i}}$ for all $i$.

Lemma 4.5. Let $F_{1}$ and $F_{2}$ be nonabelian free groups of finite rank and let $\bar{a}$ (respectively $\bar{b}$ ) be a tuple from $F_{1}$ (respectively $F_{2}$ ) such that $t p_{\exists}^{F_{1}}(\bar{a})=t p_{\exists}^{F_{2}}(\bar{b})$. Suppose that $(\bar{a}, \bar{b})$ is existentially rigid. Then either $r k\left(F_{1}\right)=2$ and $\bar{a}$ is a power of a primitive element, or there exists an embedding $h \in \operatorname{Hom}\left(F_{1}\left|\bar{a}, F_{2}\right| \bar{b}\right)$ such that $h\left(F_{1}\right)$ is an e.c. subgroup of $F_{2}$.

Proof. We assume that the first case of the conclusion of the lemma is not satisfied. Let $\varphi_{0}(\bar{x}, \bar{y})$ be the quantifier-free formula given by Proposition 4.3 applied to the tuple $(\bar{a}, \bar{b})$.

Observe that $(\bar{a}, \bar{a})$ is also existentially rigid. Hence, by Proposition 4.3 applied to the tuple $(\bar{a}, \bar{a})$, we also obtain a quantifier-free formula $\varphi_{1}(\bar{x}, \bar{y})$, such that $F_{1} \models \varphi_{1}(\bar{a}, \bar{s})$ and such that for any $f \in \operatorname{Hom}\left(F_{1}\left|\bar{a}, F_{1}\right| \bar{a}\right)$, if $F_{1} \models \varphi_{1}(\bar{a}, f(\bar{s}))$ then $f$ is an embedding.

There exists a tuple of words $\bar{w}(\bar{x})$ such that $\bar{a}=\bar{w}(\bar{s})$. Since $t p_{\exists}^{F_{1}}(\bar{a})=t p_{\exists}^{F_{2}}(\bar{b})$ we get

$$
F_{2} \models \varphi_{0}\left(\bar{b}, \bar{s}^{\prime}\right) \wedge \varphi_{1}\left(\bar{b}, \bar{s}^{\prime}\right) \wedge \bar{b}=\bar{w}\left(\bar{s}^{\prime}\right),
$$

for some tuple $\bar{s}^{\prime}$ from $F_{2}$. Since $F_{2}=\varphi_{0}\left(\bar{b}, \bar{s}^{\prime}\right) \wedge \bar{b}=\bar{w}\left(\bar{s}^{\prime}\right)$, by Proposition 4.3, the map $\bar{s} \rightarrow \bar{s}^{\prime}$ extends to an embedding sending $\bar{a}$ to $\bar{b}$, that we denote by $h$.

We claim that $h\left(F_{1}\right)$ is an e.c. subgroup of $F_{2}$. Now $h\left(F_{1}\right)$ is generated by $\bar{b}, \bar{s}^{\prime}$ and it is sufficient to show that if $\psi(\bar{x}, \bar{y})$ is an existential formula such that $F_{2} \models \psi\left(\bar{b}, \bar{s}^{\prime}\right)$ then $h\left(F_{1}\right) \models \psi\left(\bar{b}, \bar{s}^{\prime}\right)$. 
Let $\psi(\bar{x}, \bar{y})$ be an existential formula such that $F_{2} \models \psi\left(\bar{b}, \bar{s}^{\prime}\right)$. Then

$$
F_{2} \models \exists \bar{s}^{\prime}\left(\varphi_{1}\left(\bar{b}, \bar{s}^{\prime}\right) \wedge \bar{b}=\bar{w}\left(\bar{s}^{\prime}\right) \wedge \psi\left(\bar{b}, \bar{s}^{\prime}\right)\right),
$$

and since $t p_{\exists}^{F_{1}}(\bar{a})=t p_{\exists}^{F_{2}}(\bar{b})$ we get

$$
F_{1}=\varphi_{1}\left(\bar{a}, \bar{s}^{\prime \prime}\right) \wedge \bar{a}=\bar{w}\left(\bar{s}^{\prime \prime}\right) \wedge \psi\left(\bar{a}, \bar{s}^{\prime \prime}\right),
$$

for some tuple $\bar{s}^{\prime \prime}$ of $F_{1}$.

Hence the map $\bar{s} \rightarrow \bar{s}^{\prime \prime}$ extends to a monomorphism of $F_{1}$ fixing $\bar{a}$ that we denote by $h^{\prime}$.

By Proposition 2.9, if $h^{\prime}$ is not an automorphism, then $F_{1}$ is freely decomposable with respect to the subgroup generated by $\bar{a}$. Let $F_{1}=C * D$ be a nontrivial free decomposition, with $C$ containing $\bar{a}$ of minimal rank for this property. If $C$ is nonabelian, then we get a contradiction to the fact that $(\bar{a}, \bar{a})$ is existentially rigid.

Hence $C$ is abelian and, in this case, $C$ is cyclic. Therefore $\bar{a}$ is a power of a primitive element. We observe that if $r k\left(F_{1}\right)>2$, then $F_{1}$ has a nonabelian free factor containing $C$ and thus $(\bar{a}, \bar{a})$ is not existentially rigid. Therefore $r k\left(F_{1}\right)=2$; which is a contradiction of our assumption.

Thus $h^{\prime}$ is an automorphism of $F_{1}$ which fixes $\bar{a}$. Therefore $F_{1} \models \psi(\bar{a}, \bar{s})$. Since $h$ is an embedding, we get $h\left(F_{1}\right) \models \psi(h(\bar{a}), h(\bar{s}))$; that is $h\left(F_{1}\right) \models \psi\left(\bar{b}, \bar{s}^{\prime}\right)$. Therefore $h\left(F_{1}\right)$ is e.c. in $F_{2}$ as required.

Proposition 4.6. Let $F_{1}$ and $F_{2}$ be nonabelian free groups of finite rank and let $\bar{a}$ (respectively $\bar{b}$ ) be a tuple from $F_{1}$ (respectively $F_{2}$ ) such that $t p_{\exists}^{F_{1}}(\bar{a})=t p_{\exists}^{F_{2}}(\bar{b})$. Then one of the following cases holds:

(1) There exists a tuple $\bar{c}$ in $F_{1}$ which is a power of a primitive element such that $t p_{\exists}^{F_{1}}(\bar{a})=t p_{\exists}^{F_{1}}(\bar{c}) ;$

(2) There exists an e.c. subgroup $E(\bar{a})$ (respectively $E(\bar{b})$ ) containing $\bar{a}$ (respectively $\bar{b})$ of $F_{1}$ (respectively $F_{2}$ ) and an isomorphism $\tau: E(\bar{a}) \rightarrow E(\bar{b})$ sending $\bar{a}$ to $\bar{b}$.

Proof. If $(\bar{a}, \bar{b})$ is existentially rigid then the result follows from Lemma 4.5.

Let us now treat the case when $(\bar{a}, \bar{b})$ is not existentially rigid. Let $F_{1}=C * B$ be a nontrivial free decomposition and $\bar{c}$ in $C$ such that $t p_{\exists}^{C}(\bar{c})=t p_{\exists}^{F_{1}}(\bar{a})$. We may choose $C$ of minimal rank satisfying the previous property.

Suppose that $C$ is freely decomposable with respect to the subgroup generated by $\bar{c}$. Let $C=C_{1} * C_{2}$, with $\bar{c}$ in $C_{1}$. If $C_{1}$ is nonabelian then $\operatorname{tp}_{\exists}^{C_{1}}(\bar{c})=t p_{\exists}(\bar{c})$ because $C_{1} \preceq_{\exists} C$. Thus we have a contradiction with the choice of $C$ as $C_{1}$ has a smaller rank.

Thus $C_{1}$ is cyclic and thus $\bar{a}$ has the same existential type as a power of a primitive element and we get (1). 
Hence, we assume that $C$ is freely indecomposable with respect to the subgroup generated by $\bar{c}$. We see that $(\bar{c}, \bar{a})$ is existentially rigid in $C$ as otherwise we get a contradiction to the minimality of the rank of $C$.

By Lemma 4.5, there exists an embedding $h_{1}: C \rightarrow F_{1}$ such that $h_{1}(C)$ is an e.c. subgroup of $F_{1}$ and $h_{1}(\bar{c})=\bar{a}$.

Similarly $(\bar{c}, \bar{b})$ is existentially rigid and by Lemma 4.5 there exists an embedding $h_{2}: C \rightarrow F_{2}$ such that $h_{2}(C)$ is an e.c. subgroup of $F_{2}$ and $h_{2}(\bar{c})=\bar{b}$.

By setting $E(\bar{a})=h_{1}(C)$ and $E(\bar{b})=h_{2}(C), h_{2} \circ h_{1}^{-1}: E(\bar{a}) \rightarrow E(\bar{b})$ is an isomorphism with $h_{2} \circ h_{1}^{-1}(\bar{a})=\bar{b}$ and thus we get $(2)$.

Proposition 4.7. Let $F$ be a nonabelian free group of finite rank and let $\bar{a}$ and $\bar{b}$ be tuples from $F$ such that $t p_{\exists}^{F}(\bar{a})=t p_{\exists}^{F}(\bar{b})$. If $(\bar{a}, \bar{b})$ is existentially rigid then there exists an automorphism of $F$ sending $\bar{a}$ to $\bar{b}$.

Proof. By Lemma 4.5 we treat two cases. If $r k(F)=2$ and $\bar{a}$ is a power of a primitive element, then the result follows from the $\exists$-homogeneity of the free group of rank 2. The case $r k(F)=2$ with $\bar{b}$ a power of a primitive element is similar.

By Lemma 4.5, there exists a monomorphism sending $\bar{a}$ to $\bar{b}$ and a monomorphism sending $\bar{b}$ to $\bar{a}$. Hence we conclude by Lemma 3.7 .

Remark 4.8. We note that in the free group of rank 2 any tuple $(\bar{a}, \bar{b})$ is existentially rigid. Hence the above proposition can be seen as a kind of generalization of the $\exists$-homogeneity of the free group of rank 2 .

We need the following lemma in the proof of the next proposition. For the definition of Nielsen transformations we refer the reader to [13].

Lemma 4.9. If $E$ is a proper e.c. subgroup of a free group of finite rank $F$ then $r k(E)<r k(F)$.

Proof. We first claim that $E$ has finite rank. Suppose as a contradiction that $E$ has an infinite rank and let $\left\{x_{i} \mid i \in \mathbb{N}\right\}$ be a basis of $E$. Let $m$ be the rank of $F$. Since $E$ is e.c. in $F$, we conclude that for every $n$ the subgroup $L_{n}$ generated by $\left\{x_{1}, \ldots, x_{n}\right\}$ is contained in a subgroup $K_{n}$ of $E$ of rank at most $m$. But each $L_{n}$ is also a free factor of $K_{n}$; which is a contradiction for large $n$.

Hence $E$ has a finite rank $m^{\prime}$. Now, as before, $E$ is contained in a subgroup of itself of rank at most $m$. Hence $m^{\prime} \leq m$ as required.

Suppose now that $E$ is proper and suppose as a contradiction that $r k(E)=$ $r k(F)$. Let $\left\{h_{1}, \ldots, h_{m}\right\}$ be a basis of $E$ and let $\left\{x_{1}, \ldots, x_{m}\right\}$ be a basis of $F$. Then for every $i$, there exists a reduced word $w_{i}(\bar{x})$ such that $h_{i}=w_{i}(\bar{x})$. Hence in $E$, we can find $x_{1}^{\prime}, \ldots, x_{m}^{\prime}$ such that $h_{i}=w_{i}\left(\bar{x}^{\prime}\right)$. In particular, $\left\{x_{1}^{\prime}, \ldots, x_{m}^{\prime}\right\}$ is a basis of $E$. Hence, since $\left\{h_{1}, \ldots, h_{m}\right\}$ is a basis of $E$, there exists a sequence of Nielsen transformations sending $\left\{x_{1}^{\prime}, \ldots, x_{m}^{\prime}\right\}$ to $\left\{w_{1}\left(\bar{x}^{\prime}\right), \ldots, w_{m}\left(\bar{x}^{\prime}\right)\right\}$. The corresponding sequence of Nielsen transformations sends $\left\{x_{1}, \ldots, x_{m}\right\}$ to $\left\{w_{1}(\bar{x}), \ldots, w_{m}(\bar{x})\right\}$ in $F$, and thus $F$ is also generated by $\left\{h_{1}, \ldots, h_{m}\right\}$; which is a contradiction. 
Proposition 4.10. Let $F$ be a nonabelian free group of finite rank. Then the following properties are equivalent:

(1) F is $\exists$-homogeneous;

(2) The following properties are satisfied:

(i) If a tuple $\bar{a}$ is a power of a primitive element and $\bar{b}$ is of the same existential type as $\bar{a}$, then $\bar{b}$ is the power of a primitive element;

(ii) Every e.c. subgroup of $F$ is a free factor.

Proof. Suppose that (1) holds. We see that (i) is an immediate consequence. Let $E$ be an e.c. subgroup of $F$. Let $\left\{s_{1}, \ldots, s_{p}\right\}$ be a basis of $E$ and $\left\{d_{1}, \ldots, d_{q}\right\}$ be a basis of $F$. Then by Lemma $4.9, r k(E) \leq r k(F)$ and thus $p \leq q$. Let $H$ be the subgroup generated by $\left\{d_{1}, \ldots, d_{p}\right\}$. Then $H$ is an e.c. subgroup of $F$ and thus $t p_{\exists}^{F}\left(d_{1}, \ldots, d_{q}\right)=t p_{\exists}^{F}\left(s_{1}, \ldots, s_{q}\right)$. Hence by (1), there is an automorphism sending $E$ to $H$ and thus $E$ is a free factor.

Suppose that (2)(i) and (ii) hold. The case of powers of primitive elements is resolved by (i) and the other case is resolved by (ii) using Proposition 4.6.

\section{Homogeneity in Free Groups}

We are concerned in this section with homogeneity in free groups and the main purpose is to give the proof of Theorem 1.1 with the hypothesis $P=\emptyset$. The general case will be treated in the next section. We use the notation of the previous section. For a tuple $\bar{a}$ from $F$, we denote by $t p_{\exists \forall}^{F}(\bar{a})$ its $\exists \forall$-type.

Definition 5.1. Let $F$ be a nonabelian free group of finite rank and let $\bar{a}$ be a tuple of $F$. We say that $\bar{a}$ is $\forall \exists$-rigid if there is no nontrivial free decomposition $F=A * B$ such that $A$ contains a tuple $\bar{c}$ with $t p_{\exists \forall}^{F_{1}}(\bar{a})=t p_{\exists \forall}^{A}(\bar{c})$.

The first purpose is to show the following proposition, which is the analogue of Proposition 4.3. But first, we shall need to make a preliminary study of certain sequences of subgroups similar to those which appear in the proof of Proposition 4.3.

Proposition 5.2. Let $F_{1}$ and $F_{2}$ be nonabelian free groups of finite rank and let $\bar{a}$ (respectively $\bar{b})$ be a tuple from $F_{1}$ (respectively $F_{2}$ ) such that $\operatorname{tp}_{\exists \forall}^{F_{1}}(\bar{a})=t p_{\exists \forall}^{F_{2}}(\bar{b})$. Suppose that $\bar{a}$ is $\forall \exists$-rigid in $F_{1}$ and let $\bar{s}$ be a basis of $F_{1}$. Then there exists a universal formula $\varphi(\bar{x}, \bar{y})$ such that $F_{1} \models \varphi(\bar{a}, \bar{s})$ and such that for any $f \in$ $\operatorname{Hom}\left(F_{1}\left|\bar{a}, F_{2}\right| \bar{b}\right)$, if $F_{2} \models \varphi(\bar{b}, f(\bar{s}))$ then $f$ is an embedding.

Definition 5.3. Let $F$ be a free group and let $\bar{b}$ be a tuple from $F$. A sequence $\left(L_{n} \mid n \in \mathbb{N}\right)$ of subgroups of $F$ is called good if it satisfies the following properties:

(1) There exists a fixed group $D$ such that:

(i) $D$ contains $\bar{b}$;

(ii) $D$ is freely indecomposable relative to the subgroup generated by $\bar{b}$;

(iii) $D$ is a free factor of $L_{n}$ for all $n$; 
(2) There exists a fixed integer $r$ such that $r k\left(L_{n}\right)=r$ for all $n$;

(3) For any universal formula $\varphi(\bar{x}, \bar{y})$ such that $\exists \bar{y} \varphi(\bar{x}, \bar{y}) \in t p_{\exists \forall}^{F}(\bar{b})$, there exist $n \in \mathbb{N}$ and $\bar{\alpha}_{n} \in L_{n}$ such that $F \models \varphi\left(\bar{b}, \bar{\alpha}_{n}\right)$.

For such a sequence, $r$ is called the rank and $D$ is called the free factor.

Our aim now is to show the following proposition.

Proposition 5.4. Let $F$ be a nonabelian free group of finite rank and let $\bar{b}$ be a tuple from $F$. If $\left(L_{n} \mid n \in \mathbb{N}\right)$ is a good sequence then there exist $p$ and a tuple $\bar{c}$ from $L_{p}$ such that $t p_{\exists \forall}^{F}(\bar{b})=t p_{\exists \forall}^{L_{p}}(\bar{c})$.

Before proving the previous proposition, we shall need to do some preliminary work on the properties of good sequences and on the powers of primitive elements.

Lemma 5.5. Let $F$ be a nonabelian free group of finite rank and let $\bar{b}$ be a tuple from $F$. If $\left(L_{n} \mid n \in \mathbb{N}\right)$ is a good sequence, then $t p_{\exists \forall}^{F}(\bar{b}) \subseteq t p_{\exists \forall}^{L_{n}}(\bar{b})$ for all $n$.

Proof. Let $\varphi(\bar{x}, \bar{y}, \bar{z})$ be a quantifier-free formula such that $F \models \exists \bar{y} \forall \bar{z} \varphi(\bar{b}, \bar{y}, \bar{z})$.

By Definition 5.3(3), we have $F \models \forall \bar{z} \varphi(\bar{b}, \bar{\beta}, \bar{z})$ for some $p$ and a tuple $\bar{\beta}$ in $L_{p}$.

Since the previous formula is universal and $\bar{\beta}, \bar{b}$ are in $L_{p}$, we obtain $L_{p} \models$ $\forall \bar{z} \varphi(\bar{b}, \bar{\alpha}, \bar{z})$. Therefore $L_{p} \models \exists \bar{y} \forall \bar{z} \varphi(\bar{b}, \bar{y}, \bar{z})$.

By Definition 5.3(1), $L_{n}=D * C_{n}$ for all $n$ and by Definition 5.3(2) we have $r k\left(C_{n}\right)=r k\left(C_{m}\right)$ for all $n, m$.

Therefore for any $n$, there exists an isomorphism $h_{n}: L_{n} \rightarrow L_{p}$ fixing $D$ pointwise. Since $h_{n}$ is an isomorphism fixing $\bar{b}$, we get for all $n, L_{n} \models \exists \bar{y} \forall \bar{z} \varphi(\bar{b}, \bar{y}, \bar{z})$ as required.

Lemma 5.6. Let $F$ be a nonabelian free group of finite rank. If $\bar{a}$ is a power of a primitive element and $\bar{b}$ is such that $t p_{\exists}^{F}(\bar{a})=t p_{\exists}^{F}(\bar{b})$ then $t p_{\exists \forall}^{F}(\bar{a}) \subseteq t p_{\exists \forall}^{F}(\bar{b})$.

Proof. Write $\bar{a}=\left(a_{1}, \ldots, a_{q}\right)$ and $\bar{b}=\left(b_{1}, \ldots, b_{q}\right)$. First we prove

Claim 1. There exists a primitive element $u$ and an element $v$ such that:

(i) $t p_{\exists}^{F}(v)=t p_{\exists}^{F}(u)$;

(ii) There are integers $p_{1}, \ldots, p_{q}$ such that $a_{i}=u^{p_{i}}$ and $b_{i}=v^{p_{i}}$ for all $i$.

Proof. Let $u$ be a primitive element and let $p_{1}, \ldots, p_{q}$ be such that $a_{i}=u^{p_{i}}$ for all $i$. Since $t p_{\exists}^{F}(\bar{a})=t p_{\exists}^{F}(\bar{b})$, we find $v \in F$ such that $b_{i}=v^{p_{i}}$ for all $i$. Let $\varphi(x) \in t p_{\exists}^{F}(u)$. Then

$$
F \models \exists v^{\prime}\left(\varphi\left(v^{\prime}\right) \wedge_{1 \leq i \leq q} b_{i}=v^{\prime p_{i}}\right),
$$

and since $F$ is torsion-free and commutative transitive, we conclude that $v=v^{\prime}$ and thus $\varphi(x) \in t p_{\exists}^{F}(v)$. The inclusion $t p_{\exists}^{F}(v) \subseteq t p_{\exists}^{F}(u)$ can be proved using a similar argument. 
Let $\left\{x_{1}, \ldots, x_{n}\right\}$ be a basis of $F$ and let $L$ be the free group with basis $\left\{x_{1}, \ldots, x_{n}, d\right\}$. Now we show the following claim.

Claim 2. Let $u$ and $v$ be as in Claim 1. Then $t p_{\exists}^{L}(u, d)=t p_{\exists}^{L}(v, d)$.

Proof. First we show that $t p_{\exists}^{L}(u, d) \subseteq t p_{\exists}^{L}(v, d)$. Let us denote by $E(u, d)$ (respectively $E(v, d))$ the subgroup generated by $\{u, d\}$ (respectively $\{v, d\}$ ).

Let $\varphi(x, y) \in t p_{\exists}^{L}(u, d)$. Since $E(u, d)$ is a free factor of $L$, it is an e.c. subgroup of $L$ and thus $E(u, d) \models \varphi(u, d)$. Since $E(u, d)$ and $E(v, d)$ are isomorphic by the map sending $u$ to $v$ and $d$ to itself, we conclude that $E(v, d) \models \varphi(v, d)$ and therefore $L \models \varphi(v, d)$ as required.

Now we show that $t p_{\exists}^{L}(v, d) \subseteq t p_{\exists}^{L}(u, d)$. Let $\varphi(x, y) \in t p_{\exists}^{L}(v, d)$. Then $\varphi(x, y)$ can be written as

$$
\exists \bar{z} \bigvee_{1 \leq i \leq p}\left(\bigwedge_{W \in P_{i}} W(x, y, \bar{z})=1 \wedge \bigwedge_{V \in N_{i}} V(x, y, \bar{z}) \neq 1\right),
$$

where $P_{i}$ and $N_{i}$ are finite for all $i$. Hence there is a tuple of words $\bar{\alpha}(\bar{x}, t)$ and $q$ such that

$$
L \models \bigwedge_{W \in P_{q}} W(v, d, \bar{\alpha}(\bar{x}, d))=1 \wedge \bigwedge_{V \in N_{q}} V(v, d, \bar{\alpha}(\bar{x}, d)) \neq 1 .
$$

Now we make the following observation. Let $v(\bar{x})$ be a reduced word such that $v=v(\bar{x})$ in $F$. Then $L$ can be viewed as the group with the generating set $\left\{x_{1}, \ldots, x_{n}, d, v\right\}$ and with the presentation $v=v(\bar{x})$. Hence in any group $G$ with a generating set $\left\{x_{1}^{\prime}, \ldots, x_{n}^{\prime}, d_{0}, v_{0}\right\}$ satisfying $v_{0}=v\left(\bar{x}^{\prime}\right)$ we get

$$
\bigwedge_{W \in P_{q}} W\left(v_{0}, d_{0}, \bar{\alpha}\left(\bar{x}^{\prime}, d_{0}\right)\right)=1 .
$$

Since $F$ is an e.c. subgroup of $L$ and since $t p_{\exists}^{F}(v)=t p_{\exists}^{F}(u)$, we find $\bar{x}^{\prime}, d^{\prime} \in F$ such that

$$
F \models \bigwedge_{W \in P_{q}} W\left(u, d^{\prime}, \bar{\alpha}\left(\bar{x}^{\prime}, d^{\prime}\right)\right)=1 \wedge \bigwedge_{V \in N_{q}} V\left(u, d^{\prime}, \bar{\alpha}\left(\bar{x}^{\prime}, d^{\prime}\right)\right) \neq 1 \wedge u=v\left(\bar{x}^{\prime}\right) .
$$

Let $G$ be the subgroup of $L$ generated by $\left\{x_{1}^{\prime}, \ldots, x_{n}^{\prime}, d, u\right\}$. Since $u=v\left(\bar{x}^{\prime}\right)$, we get by the above observation and by replacing $v_{0}$ by $u$ and $d_{0}$ by $d$ that

$$
L \models \bigwedge_{W \in P_{q}} W\left(u, d, \bar{\alpha}\left(\bar{x}^{\prime}, d\right)\right)=1 .
$$

Let $f: L \rightarrow F$ be the homomorphism fixing pointwise $F$ and sending $d$ to $d^{\prime}$. Since

$$
F \models \bigwedge_{V \in N_{q}} V\left(u, f(d), \bar{\alpha}\left(\bar{x}^{\prime}, f(d)\right)\right) \neq 1
$$

we conclude that

$$
L \models \bigwedge_{V \in N_{q}} V\left(u, d, \bar{\alpha}\left(\bar{x}^{\prime}, d\right)\right) \neq 1
$$


By (5.1) and (5.2), we conclude that

$$
L \models \bigwedge_{W \in P_{q}} W\left(u, d, \bar{\alpha}\left(\bar{x}^{\prime}, d\right)\right)=1 \wedge \bigwedge_{V \in N_{q}} V\left(u, d, \bar{\alpha}\left(\bar{x}^{\prime}, d\right)\right) \neq 1
$$

and finally $L \models \exists \bar{z}\left(\bigwedge_{W \in P_{q}} W(u, d, \bar{z})=1 \wedge \bigwedge_{V \in N_{q}} V(u, d, \bar{z}) \neq 1\right)$.

Thus $t p_{\exists}^{L}(v, d) \subseteq t p_{\exists}^{L}(u, d)$ as required and this ends the proof of the claim.

Claim 3. Let $u$ and $v$ be as in Claim 1. Then $t p_{\exists \forall}^{L}(u, d) \subseteq t p_{\exists \forall}^{L}(v, d)$.

Proof. Let us denote by $E(u, d)$ (respectively $E(v, d))$ the subgroup generated by $\{u, d\}$ (respectively $\{v, d\}$ ). By Claim $1, t p_{\exists}^{L}(u, d)=t p_{\exists}^{L}(v, d)$ and since $E(u, d)$ is an e.c. subgroup of $L$, we conclude that $E(v, d)$ is e.c. in $L$.

Since $E(u, d)$ is an elementary subgroup of $L$ we have $t p_{\exists \forall}^{L}(u, d)=t p_{\exists \forall}^{E(u, d)}(u, d)$. Since $E(u, d)$ and $E(v, d)$ are isomorphic by the map sending $u$ to $v$ and fixing $d$, we conclude that $t p_{\exists \forall}^{E(u, d)}(u, d)=t p_{\exists \forall}^{E(v, d)}(v, d)$. Therefore $t p_{\exists \forall}^{L}(u, d)=t p_{\exists \forall}^{E(v, d)}(v, d)$.

Now since $E(v, d)$ is e.c. in $L$ we get $t p_{\exists \forall}^{E(v, d)}(v, d) \subseteq t p_{\exists \forall}^{L}(v, d)$ and finally $t p_{\exists \forall}^{L}(u, d) \subseteq t p_{\exists \forall}^{L}(v, d)$ as required.

Let $u$ and $v$ be as in Claim 1. It follows by Claim 3 that $t p_{\exists \forall}^{L}(u) \subseteq t p_{\exists \forall}^{L}(v)$. Since $F$ is an elementary subgroup of $L$, we conclude that $t p_{\exists \forall}^{F}(u) \subseteq t p_{\exists \forall}^{F}(v)$.

Now let us show that $t p_{\exists \forall}^{F}(\bar{a}) \subseteq t p_{\exists \forall}^{F}(\bar{b})$. Let $p_{1}, \ldots, p_{q}$ be given by Claim 1. Let $\varphi\left(x_{1}, \ldots, x_{q}\right) \in t p_{\exists \forall}^{F}(\bar{a})$. Then $\varphi\left(x^{p_{1}}, \ldots, x^{p_{q}}\right) \in t p_{\exists \forall}^{F}(u)$. Since $t p_{\exists \forall}^{F}(u) \subseteq t p_{\exists \forall}^{F}(v)$ we conclude that $\varphi\left(x^{p_{1}}, \ldots, x^{p_{q}}\right) \in t p_{\exists \forall}^{F}(v)$ and hence $\varphi\left(x_{1}, \ldots, x_{q}\right) \in t p_{\exists \forall}^{F}(\bar{b})$. This ends the proof of the lemma.

Having disposed of this preliminary step, we are now in a position to prove Proposition 5.4.

Proof of Proposition 5.4. The proof is by induction on the rank of good sequences. Let $\left(L_{n} \mid n \in \mathbb{N}\right)$ be a good sequence of $F$, let $r$ be its rank and let $D$ be its free factor. Let $\bar{s}$ be a basis of $F$. Let $(*)$ be the following property:

(*) for any universal formula $\varphi(\bar{x})$ such that $F \models \varphi(\bar{s})$, there exists $f \in$ $\operatorname{Hom}(F|\bar{b}, F| \bar{b})$ such that $F \models \varphi(f(\bar{s}))$ where $f$ is non-injective in restriction to $D$.

We are going to handle two cases according to whether $(*)$ is satisfied or not. Let us first treat the case when $(*)$ holds.

Claim 1. There exists a sequence $\left(H_{p} \mid p \in \mathbb{N}\right)$ satisfying the following properties:

(i) For any $p \in \mathbb{N}$, there exist $n \in \mathbb{N}$ and $f \in \operatorname{Hom}(F|\bar{b}, F| \bar{b})$ such that $H_{p}=f\left(L_{n}\right)$ and such that $f$ is non-injective in restriction to $L_{n}$;

(ii) For any universal formula $\varphi(\bar{x}, \bar{y})$ such that $\exists \bar{y} \varphi(\bar{x}, \bar{y}) \in t p_{\forall \exists}^{F}(\bar{b})$, there exists $p_{0}$ such that for any $p \geq p_{0}$ there exists $\bar{\beta}_{p}$ in $H_{p}$ such that $F \models \varphi\left(\bar{b}, \bar{\beta}_{p}\right)$. 
Proof. Let $\left(\psi_{i}\left(\bar{x}, \bar{y}_{i}\right) \mid i \in \mathbb{N}\right)$ be an enumeration of the universal formulas such that $\exists \bar{y}_{i} \psi_{i}\left(\bar{x}, \bar{y}_{i}\right) \in t p_{\forall}^{F}(\bar{s})$ and, for every $n \in \mathbb{N}$, let

$$
\varphi_{n}\left(\bar{x}, \bar{y}_{0}, \ldots, \bar{y}_{n}\right)=\bigwedge_{0 \leq i \leq n} \psi_{i}\left(\bar{x}, \bar{y}_{i}\right) .
$$

We define $\left(H_{p} \mid p \in \mathbb{N}\right)$ as follows. Let $p \in \mathbb{N}$. Since $\left(L_{n} \mid n \in \mathbb{N}\right)$ is good, by (3) of Definition 5.3 , there exists $n_{p} \in \mathbb{N}$ such that for some sequence $\left(\bar{\alpha}_{0}, \ldots, \bar{\alpha}_{p}\right)$ in $L_{n_{p}}$,

$$
F \models \varphi_{p}\left(\bar{b}, \bar{\alpha}_{0}, \ldots, \bar{\alpha}_{p}\right) .
$$

By $(*)$, there exists a homomorphism $f \in \operatorname{Hom}(F|\bar{b}, F| \bar{b})$ such that

$$
F \models \varphi_{p}\left(\bar{b}, f\left(\bar{\alpha}_{0}\right), \ldots, f\left(\bar{\alpha}_{p}\right)\right)
$$

which is not injective in restriction to $D$. In particular, $f$ is non-injective in restriction to $L_{n_{p}}$.

Put $H_{p}=f\left(L_{n_{p}}\right)$. Thus we get (i). We note that $\left(f\left(\bar{\alpha}_{0}\right), \ldots, f\left(\bar{\alpha}_{p}\right)\right)$ is a sequence of $H_{p}$. By construction we have (ii).

We notice that by construction any subsequence $\left(H_{p_{k}} \mid k \in \mathbb{N}\right)$ also satisfies (i) and (ii) of Claim 1.

By (i), $\bar{b} \in H_{p}=f\left(L_{q}\right)$ for some $q$, and since $f$ is not injective in restriction to $D$ we have $r k\left(H_{p}\right)<r k\left(L_{q}\right)=r$ for all $p$. Proceeding as in the proof of Proposition 4.3, by using the pigeon hole principle we extract a subsequence, which we assume to simplify the notation to be $\left(H_{p} \mid p \in \mathbb{N}\right)$ itself, such that $r k\left(H_{p}\right)$ is a fixed natural number $r^{\prime}<r$ for all $p$.

Again, proceeding as in the proof of Proposition 4.3, and up to extracting a subsequence, we may assume that for all $p \in \mathbb{N}$,

$$
H_{p}=\left\langle h_{1}, \ldots, h_{q}, h_{(q+1) p}, \ldots, h_{r p}\right\rangle
$$

and $\bar{b}$ is in the subgroup with basis $\left\{h_{1}, \ldots, h_{q}\right\}$.

Let $H$ be the subgroup with basis $\left\{h_{1}, \ldots, h_{q}\right\}$. By the Grushko decomposition, we have $H=M * N$ where $\bar{b}$ is in $M$ and $M$ is freely indecomposable with respect to the subgroup generated by $\bar{b}$. We define $M$ to be the free factor of the sequence and thus we get (1) of Definition 5.3. By construction, the sequence $\left(H_{p} \mid p \in \mathbb{N}\right)$ satisfies (2) and (3) of Definition 5.3 and hence is a good sequence.

By induction, there exists $p$ such that $H_{p}$ has a tuple $\bar{c}$ with $t p_{\exists \forall}^{F}(\bar{b})=t p_{\exists \forall}^{H_{p}}(\bar{c})$. Now by construction, there exists $q$ and $f \in \operatorname{Hom}(F|\bar{b}, F| \bar{b})$ such that $H_{p}=f\left(L_{q}\right)$ and $f$ is non-injective in restriction to $L_{q}$. By Proposition 2.9, $L_{q}$ has a free decomposition $L_{q}=A * B$ such that $f(A)=H_{p}$ and $f(B)=1$ and where $f$ is injective in restriction to $A$. Since $f$ is injective in restriction to $A, A$ contains a tuple $\bar{c}^{\prime}$ such that $t p_{\exists \forall}^{H_{p}}(\bar{c})=t p_{\exists \forall}^{A}\left(\bar{c}^{\prime}\right)$. Since $A$ is an elementary subgroup of $L_{p}$ and $t p_{\exists \forall}^{F}(\bar{b})=t p_{\exists \forall}^{H_{p}}(\bar{c})$, we conclude that $t p_{\exists \forall}^{L_{q}}\left(\bar{c}^{\prime}\right)=t p_{\exists \forall}^{F}(\bar{b})$. This ends the proof when (*) is satisfied. 
We treat now the case when $(*)$ is not true. We treat the two cases depending on whether $D$ is abelian or not.

Suppose that $D$ is abelian. Hence $D$ is cyclic and we assume that it is generated by $u$. Write $\bar{b}=\left(b_{1}, \ldots, b_{q}\right)$ and let $p_{1}, \ldots, p_{q}$ integers such that $b_{i}=u^{p_{i}}$ for all $i$.

Let $u^{\prime}$ be a primitive element in $F$ and let $\bar{b}^{\prime}=\left(u^{\prime p_{1}}, \ldots, u^{\prime p_{q}}\right)$. By Theorem 2.4, we conclude that $t p_{\exists \forall}^{L_{n}}(\bar{b})=t p_{\exists \forall}^{F}\left(\bar{b}^{\prime}\right)$.

By Lemma 5.5, $t p_{\exists \forall}^{F}(\bar{b}) \subseteq t p_{\exists \forall}^{L_{n}}(\bar{b})$ for all $n$. Therefore $t p_{\exists \forall}^{F}(\bar{b}) \subseteq t p_{\exists \forall}^{F}\left(\bar{b}^{\prime}\right)$. In particular $t p_{\exists}^{F}(\bar{b})=t p_{\exists}^{F}\left(\bar{b}^{\prime}\right)$. By Lemma 5.6, we get $t p_{\exists \forall}^{F}(\bar{b})=t p_{\exists \forall}^{F}\left(\bar{b}^{\prime}\right)$.

By $t p_{\exists \forall}^{L_{n}}(\bar{b})=t p_{\exists \forall}^{F}\left(\bar{b}^{\prime}\right)$, we conclude that $t p_{\exists \forall}^{L_{n}}(\bar{b})=t p_{\exists \forall}^{F}(\bar{b})$. Hence in this case we get the required result.

Suppose now that $D$ is nonabelian. Since $(*)$ is not true, there exists a universal formula $\varphi_{0}(\bar{x})$ such that $F \models \varphi(\bar{s})$ and such that for any $f \in \operatorname{Hom}(F|\bar{b}, F| \bar{b})$ if $F \models \varphi_{0}(f(\bar{s}))$ then $f$ is injective in restriction to $D$.

We claim that $D$ is e.c. in $F$. Let $\bar{d}$ be a basis of $D$. Then there exists a tuple of words $\bar{w}(\bar{y})$ such that $\bar{d}=\bar{w}(\bar{s})$ and a tuple of words $\bar{v}(\bar{y})$ such that $\bar{b}=\bar{v}(\bar{d})$.

Let $\varphi(\bar{x}, \bar{y}, \bar{z})$ be a quantifier-free formula such that $F \models \exists \bar{z} \varphi(\bar{b}, \bar{d}, \bar{z})$.

Thus

$$
F \models \exists \bar{z} \exists \bar{d} \exists \bar{s}\left(\varphi(\bar{b}, \bar{d}, \bar{z}) \wedge \varphi_{0}(\bar{s}) \wedge \bar{d}=\bar{w}(\bar{s}) \wedge \bar{b}=\bar{v}(\bar{d})\right)
$$

Since $\left(L_{n} \mid n \in \mathbb{N}\right)$ is a good sequence, there exist $p$ and tuples of elements of $L_{p}, \bar{\alpha}, \bar{d}^{\prime}, \bar{s}^{\prime}$ such that

$$
F \models \varphi\left(\bar{b}, \bar{d}^{\prime}, \bar{\alpha}\right) \wedge \varphi_{0}\left(\bar{s}^{\prime}\right) \wedge \bar{d}^{\prime}=\bar{w}\left(\bar{s}^{\prime}\right) \wedge \bar{b}=\bar{v}\left(\bar{d}^{\prime}\right) .
$$

Hence the homomorphism $f$ which sends $\bar{s}$ to $\bar{s}^{\prime}$ is injective on $D$ and fixes $\bar{b}$.

Let $D^{\prime}$ be the subgroup of $L_{p}$ generated by $\bar{d}^{\prime}$. Using the Grushko decomposition and since $\bar{b}$ is in $D \cap D^{\prime}$ and since $D$ is freely indecomposable relative to the subgroup generated by $\bar{b}$, we conclude that $D^{\prime} \leq D$. Therefore the map $\bar{d} \rightarrow \bar{d}^{\prime}$ extends to a monomorphism $h$ of $D$ fixing $\bar{b}$. Since $D$ is freely indecomposable relative to the subgroup generated by $\bar{b}$, by Proposition $2.10, h$ is an automorphism of $D$. Since $D$ is a free factor of $L_{n}, h$ can be extended to an automorphism of $L_{n}$ that we still denote by $h$.

Since

$$
L_{n} \models \exists \bar{z} \varphi(\bar{b}, h(\bar{d}), \bar{z}),
$$

we conclude that

$$
L_{n} \models \exists \bar{z} \varphi(\bar{b}, \bar{d}, \bar{z})
$$

and thus $D \models \exists \bar{z} \varphi(\bar{b}, \bar{d}, \bar{z})$ as $D$ is e.c. in $L_{n}$.

Hence $D$ is an e.c. subgroup of $F$ as claimed. Thus $t p_{\exists \forall}^{D}(\bar{b}) \subseteq t p_{\exists \forall}^{F}(\bar{b})$ and, since $D$ is an elementary subgroup of $L_{n}$, we get $t p_{\exists \forall}^{L_{n}}(\bar{b})=t p_{\exists \forall}^{D}(\bar{b})$. Therefore $t p_{\exists \forall}^{L_{n}}(\bar{b}) \subseteq t p_{\exists \forall}^{F}(\bar{b})$ and $t p_{\exists \forall}^{L_{n}}(\bar{b})=t p_{\exists \forall}^{F}(\bar{b})$ by Lemma 5.5 . This ends the proof of the proposition. 
Proof of Proposition 5.2. The proof proceeds in a similar way to that of Proposition 4.3. Let $\left(\psi_{i}(\bar{x}, \bar{y}) \mid i \in \mathbb{N}\right)$ be an enumeration of $t p_{\forall}^{F_{1}}(\bar{a}, \bar{s})$ and set

$$
\varphi_{n}(\bar{x}, \bar{y})=\wedge_{0 \leq i \leq n} \psi_{i}(\bar{x}, \bar{y}) .
$$

Suppose as a contradiction that for any $n \in \mathbb{N}$, there exists a non-injective homomorphism $f_{n} \in \operatorname{Hom}\left(F_{1}\left|\bar{a}, F_{2}\right| \bar{b}\right)$ such that $F_{2} \models \varphi_{n}\left(\bar{b}, f_{n}(\bar{s})\right)$.

Observe that for any subsequence $\left(f_{n_{k}}\right)_{k \in \mathbb{N}}$ and for any $n \in \mathbb{N}$, there exists $n_{k}$ such that for any $k^{\prime} \geq k$ we have $F_{2}=\varphi_{n}\left(\bar{b}, f_{n_{k^{\prime}}}(\bar{s})\right)$.

We have $\bar{b} \in f_{n}\left(F_{1}\right)$ and, since $f_{n}$ is not injective, we have $r k\left(f_{n}\left(F_{1}\right)\right)<r k\left(F_{1}\right)$ for all $n$. Using the pigeon hole principle, we extract a subsequence, that we assume to simplify notation to be $\left(f_{n}\right)_{n \in \mathbb{N}}$ itself, such that $r k\left(f_{n}\left(F_{1}\right)\right)$ is a fixed natural number $r$ for all $n$.

Proceeding as in the proof of Proposition 4.3, and up to extracting a subsequence, we may assume that for all $n \in \mathbb{N}$,

$$
f_{n}\left(F_{1}\right)=\left\langle d_{1}, \ldots, d_{p}, d_{(p+1) n}, \ldots, p_{r n}\right\rangle
$$

and $\bar{b}$ is in the subgroup with basis $\left\{d_{1}, \ldots, d_{p}\right\}$.

Set $L_{n}=\left\langle d_{1}, \ldots, d_{p}, d_{(p+1) n}, \ldots, d_{r n}\right\rangle=f_{n}\left(F_{1}\right)$. Let $H$ be the subgroup with basis $\left\{d_{1}, \ldots, d_{q}\right\}$. By the Grushko decomposition, $H=D * N$ where $\bar{b}$ is in $D$ and $D$ is freely indecomposable with respect to the subgroup generated by $\bar{b}$.

We claim now that the sequence $\left(L_{n} \mid n \in \mathbb{N}\right)$ is a good sequence. By construction, $\left(L_{n} \mid n \in \mathbb{N}\right)$ satisfes (1) and (2) of Definition 5.3 and it remains to show (3) of the same definition.

Let $\varphi(\bar{x}, \bar{y})$ be a universal formula such that $F_{2} \models \exists \bar{y} \varphi(\bar{b}, \bar{y})$. Since $t p_{\exists \forall}^{F_{1}}(\bar{a})=$ $t p_{\exists \forall}^{F_{2}}(\bar{b})$, we get

$$
F_{1} \models \exists \bar{y} \varphi(\bar{a}, \bar{y})
$$

Therefore there exists a tuple of words $\bar{\alpha}(\bar{t})$ such that $F_{1} \models \varphi(\bar{a}, \bar{\alpha}(\bar{s}))$. By construction of the sequence $\left(f_{n}\right)_{n \in \mathbb{N}}$ we have $F_{2}=\varphi\left(\bar{b}, \bar{\alpha}\left(f_{n}(\bar{s})\right)\right)$ for all but finitely many $n$.

Therefore for large $n$ we have a tuple $\bar{\alpha}_{n}=\alpha\left(f_{n}(\bar{s})\right)$ in $L_{n}$ such that $F_{2} \models$ $\varphi\left(\bar{b}, \bar{\alpha}_{n}\right)$ and thus we get $(3)$ of Definition 5.3 .

We conclude that $\left(L_{n} \mid n \in \mathbb{N}\right)$ is a good sequence as claimed. By Proposition 5.4 , there exists $p$ and a tuple $\bar{c}$ from $L_{p}$ such that $t p_{\exists \forall}^{F_{2}}(\bar{b})=t p_{\exists \forall}^{L_{p}}(\bar{c})$.

By Proposition 2.9, $F_{1}$ has a free decomposition $F_{1}=A * B$ such that $f_{p}(A)=L_{p}$ and $f(B)=1$ and where $f_{p}$ is injective in restriction to $A$. Since $f_{p}$ is not injective, the above decomposition is nontrivial.

Thus $A$ has a tuple $\bar{c}^{\prime}$ with $t p_{\exists \forall}^{F_{2}}(\bar{b})=t p_{\exists \forall}^{A}\left(\bar{c}^{\prime}\right)$. Since $A$ is an elementary subgroup of $F_{1}$ and since $t p_{\exists \forall}^{F_{2}}(\bar{b})=t p_{\exists \forall}^{F_{1}}(\bar{a})$, we conclude that $\bar{a}$ is not $\forall \exists$-rigid, which is our final contradiction. This ends the proof of the proposition.

The following proposition is the analogue of Lemma 4.5.

Proposition 5.7. Let $F_{1}$ and $F_{2}$ be nonabelian free groups of finite rank and let $\bar{a}($ respectively $\bar{b})$ be a tuple from $F_{1}$ (respectively $\left.F_{2}\right)$ such that $\operatorname{tp}_{\exists \forall}^{F_{1}}(\bar{a})=t p_{\exists \forall}^{F_{2}}(\bar{b})$. 
Suppose that $\bar{a}$ is $\forall \exists$-rigid. Then either $r k\left(F_{1}\right)=2$ and $\bar{a}$ is a power of a primitive element, or there exists an embedding $h \in \operatorname{Hom}\left(F_{1}\left|\bar{a}, F_{2}\right| \bar{b}\right)$ such that $h\left(F_{1}\right) \preceq_{\exists \forall} F_{2}$.

Proof. We suppose that the first case of the conclusion of the proposition is not satisfied. Let $\varphi_{0}(\bar{x}, \bar{y})$ be the universal formula given by Proposition 5.2 applied to the tuple $(\bar{a}, \bar{b})$.

By Proposition 5.2 applied to the tuple $(\bar{a}, \bar{a})$, we also get a universal formula $\varphi_{1}(\bar{x}, \bar{y})$ such that $F_{1} \models \varphi_{1}(\bar{a}, \bar{s})$ and such that for any $f \in \operatorname{Hom}\left(F_{1}\left|\bar{a}, F_{1}\right| \bar{a}\right)$ if $F_{1} \models \varphi_{1}(\bar{a}, f(\bar{s}))$ then $f$ is an embedding.

There exists a tuple of words $\bar{w}(\bar{x})$ such that $\bar{a}=\bar{w}(\bar{s})$. Since $t p_{\exists \forall}^{F_{1}}(\bar{a})=t p_{\exists \forall}^{F_{2}}(\bar{b})$ we get

$$
F_{2} \models \varphi_{0}\left(\bar{b}, \bar{s}^{\prime}\right) \wedge \varphi_{1}\left(\bar{b}, \bar{s}^{\prime}\right) \wedge \bar{b}=\bar{w}\left(\bar{s}^{\prime}\right),
$$

for some tuple $\bar{s}^{\prime}$ in $F_{2}$. By Proposition 5.2, the map $\bar{s} \rightarrow \bar{s}^{\prime}$ extends to an embedding that we denote by $h$.

We claim that $h\left(F_{1}\right) \preceq_{\exists \forall} F_{2}$. Let $\psi(\bar{x}, \bar{y})$ be $\exists \forall$-formula such that $F_{2}=\psi\left(\bar{b}, \bar{s}^{\prime}\right)$. Then

$$
F_{2} \models \exists \bar{s}^{\prime}\left(\varphi_{1}\left(\bar{b}, \bar{s}^{\prime}\right) \wedge \bar{b}=\bar{w}\left(\bar{s}^{\prime}\right) \wedge \psi\left(\bar{b}, \bar{s}^{\prime}\right)\right),
$$

and since $t p_{\exists \forall}^{F_{1}}(\bar{a})=t p_{\exists \forall}^{F_{2}}(\bar{b})$, we get

$$
F_{1} \models \varphi_{1}\left(\bar{a}, \bar{s}^{\prime \prime}\right) \wedge \bar{a}=\bar{w}\left(\bar{s}^{\prime \prime}\right) \wedge \psi\left(\bar{a}, \bar{s}^{\prime \prime}\right),
$$

for some tuple $\bar{s}^{\prime \prime}$ of $F_{1}$.

Hence the map $\bar{s} \rightarrow \bar{s}^{\prime \prime}$ extends to a monomorphism of $F_{1}$ fixing $\bar{a}$ that we denote by $h^{\prime}$.

By Proposition 2.9, if $h^{\prime}$ is not an automorphism then $F_{1}$ is freely decomposable with respect to the subgroup generated by $\bar{a}$. Let $F_{1}=C * D$ be a nontrivial free decomposition with $r k(C)$ of minimal rank such that $\bar{a}$ is in $C$. If $C$ is nonabelian, then we get a contradiction of the fact that $\bar{a}$ is $\forall \exists$-rigid.

Hence $C$ is abelian and in this case $D$ is cyclic and thus $\bar{a}$ is a power of a primitive element and $r k\left(F_{1}\right)=2$; which is a contradiction of our assumption.

Thus $h^{\prime}$ is an automorphism of $F_{1}$ which fixes $\bar{a}$. Therefore $F_{1} \models \psi(\bar{a}, \bar{s})$.

Since $h$ is an embedding, we get $h\left(F_{1}\right)=\psi\left(\bar{b}, \bar{s}^{\prime}\right)$. Therefore $h\left(F_{1}\right) \preceq \exists \forall F_{2}$ as required.

We now give the proof of Theorem 1.1, with the hypothesis $P=\emptyset$.

Proposition 5.8. Let $F$ be a nonabelian free group of finite rank and let $\bar{a}$ and $\bar{b}$ be tuples of $F$ such that $t p^{F}(\bar{a})=t p^{F}(\bar{b})$. Then there exists an automorphism $\sigma$ of $F$ such that $\sigma(\bar{a})=\bar{b}$.

Proof. We may assume that $r k(F)>2$. Suppose that $\bar{a}$ is $\forall \exists$-rigid. It follows in particular that $\bar{b}$ is also $\forall \exists$-rigid. By Proposition 5.7 , there exists a monomorphism 
sending $\bar{a}$ to $\bar{b}$ and a monomorphism sending $\bar{b}$ to $\bar{a}$. Hence, we conclude with Lemma 3.7.

We treat now the case where $\bar{a}$ is not $\forall \exists$-rigid. Let $F_{1}=C * B$ be a nontrivial free decomposition and $\bar{c}$ in $C$ such that $t p_{\exists \forall}^{C}(\bar{c})=t p_{\exists \forall}^{F_{1}}(\bar{a})$. We may choose $C$ to be of minimal rank satisfying the previous property.

Suppose that $C$ is freely decomposable with respect to the subgroup generated by $\bar{c}$. Let $C=C_{1} * C_{2}$ where $\bar{c}$ is in $C_{1}$. If $C_{1}$ is nonabelian then $t p_{\exists \forall}^{C_{1}}(\bar{c})=t p_{\exists \forall}^{C}(\bar{c})$, because $C_{1} \preceq_{\exists \forall} C$ by Theorem 2.4. Thus we have a contradiction with the choice of $C$ as $C_{1}$ has a smaller rank.

Thus $C_{1}$ is cyclic and thus $\bar{a}$ has the same $\exists \forall$-type as the power of a primitive element. By Theorem 2.4, we conclude that $\bar{a}$ has the same type as a power of a primitive element and, by Theorem 2.7, we get the required conclusion.

Hence, we assume that $C$ is freely indecomposable with respect to the subgroup generated by $\bar{c}$. We see that $\bar{c}$ is $\forall \exists$-rigid in $C$ as otherwise we get a contradiction of the minimality of the rank of $C$.

By Proposition 5.7, there exists an embedding $h_{1}: C \rightarrow F$ such that $h_{1}(C) \preceq_{\exists \forall}$ $F$ and $h_{1}(\bar{c})=\bar{a}$.

Similarly, by Proposition 5.7, there exists an embedding $h_{2}: C \rightarrow F$ such that $h_{2}(C) \preceq_{\exists \forall} F$ and $h_{2}(\bar{c})=\bar{b}$.

We have $h_{2} \circ h_{1}^{-1}: h_{1}(C) \rightarrow h_{2}(C)$ is an isomorphism with $h_{2} \circ h_{1}^{-1}(\bar{a})=\bar{b}$. Since $h_{2}(C) \preceq_{\exists \forall} F$ and $h_{1}(C) \preceq_{\exists \forall} F$ they are free factors of $F$ by Theorem 2.4 and Theorem 2.6. Therefore $h_{2} \circ h_{1}^{-1}$ can be extended to an automorphism of $F$ as required, because $h_{1}(C)$ and $h_{2}(C)$ have the same rank.

We conclude this section with the following proposition of independent interest.

Proposition 5.9. Let $F$ be a nonabelian free group of finite rank and let $\bar{a}$ be a tuple of $F$ such that $F$ is freely indecomposable relative to the subgroup generated by $\bar{a}$. Let $\bar{s}$ be a basis of $F$. Then there exists a universal formula $\varphi(\bar{x})$ such that $F \models \varphi(\bar{s})$ and such that for any endomorphism $f$ of $F$, if $F \models \varphi(f(\bar{s}))$ and $f$ fixes $\bar{a}$, then $f$ is an automorphism. In particular, $(F, \bar{a})$ is a prime model of the theory $\operatorname{Th}(F, \bar{a})$.

Proof. We claim that $\bar{a}$ is $\forall \exists$-rigid. Suppose as a contradiction that $\bar{a}$ is not $\forall \exists$ rigid and let $F=A * B$ be a nontrivial free decomposition such that $A$ contains a tuple $\bar{c}$ with $t p_{\exists \forall}^{F}(\bar{a})=t p_{\exists \forall}^{F}(\bar{c})$. By Theorem 2.4, we conclude that $t p^{F}(\bar{a})=t p^{F}(\bar{c})$, and by Proposition 5.8, there is an automorphism $\sigma$ sending $\bar{c}$ to $\bar{a}$. Hence $\sigma(A)$ is a free factor containing $\bar{a}$ and thus $F$ is freely indecomposable relative to the subgroup generated by $\bar{a}$; which is a contradiction to the hypothesis of the proposition.

Hence, by Proposition 5.2, there exists a universal formula $\varphi(\bar{y})$ such that $F \models$ $\varphi(\bar{s})$ and such that for any $f \in \operatorname{Hom}(F|\bar{a}, F| \bar{a})$, if $F \models \varphi(f(\bar{s}))$ then $f$ is an embedding and by Proposition 2.10, we conclude that $f$ is an automorphism.

Now the proof of the fact that $(F, \bar{a})$ is a prime model of the theory $\operatorname{Th}(F, \bar{a})$ proceeds in a similar way to that of Lemma 3.5. 


\section{Dealing with Parameters}

In this section, we show Theorems 1.1 and 1.3 for arbitrary $P$. We reduce the problem to Propositions 4.6 and 5.8 by using definable closure and existential definable closure. We recall the following definition.

Definition 6.1. Let $G$ be a group and $P \subseteq G$. The definable closure (respectively existential definable closure) of $A$, denoted $d c l(P)$ (respectively $d c l^{\exists}(P)$ ), is the set of elements $g \in G$ such that there exists a formula (respectively an existential formula) $\phi(x)$ with parameters from $P$ such that $G \models \phi(g)$ and $g$ is the unique element satisfying $\phi$.

We see that for any $P \subseteq G, d c l(P)$ and $d c l^{\exists}(P)$ are subgroups of $G$. In a forthcoming paper [23], we answer a question by Sela about definable and algebraic closure. We will use the following theorem of that paper.

Theorem 6.2. ([23]) Let $F$ be a nonabelian free group of finite rank and let $P \subseteq F$. Then $d c l(P)$ and $d c l^{\exists}(P)$ are finitely generated and their rank is bounded by the rank of $F$.

Now we show the following simple lemma.

Lemma 6.3. Let $G$ be a group and $P \subseteq G$. Let $\bar{a}$ and $\bar{b}$ be tuples from $G$.

(1) If $t p^{G}(\bar{a} \mid P)=t p^{G}(\bar{b} \mid P)$, then $t p^{G}(\bar{a} \mid \operatorname{dcl}(P))=t p^{G}(\bar{b} \mid \operatorname{dcl}(P))$.

(2) Similarly if $\operatorname{tp}_{\exists}^{G}(\bar{a} \mid P)=\operatorname{tp}_{\exists}^{G}(\bar{b} \mid P)$ then $\operatorname{tp}_{\exists}^{G}\left(\bar{a} \mid \operatorname{dcl}^{\exists}(P)\right)=\operatorname{tp}_{\exists}^{G}\left(\bar{b} \mid \operatorname{dcl}^{\exists}(P)\right)$.

Proof. (1) Let $\psi\left(\bar{x} ; y_{1}, \ldots, y_{n}\right)$ be a formula such that $\psi\left(\bar{x} ; d_{1}, \ldots, d_{n}\right) \in$ $t p^{G}(\bar{a} \mid d c l(P))$ where $d_{i} \in \operatorname{dcl}(P)$ for all $i$. For every $i$, there exists a formula $\phi_{i}(y)$ with parameters from $P$ such that $d_{i}$ is the unique element satisfying $\phi_{i}$. Since

$$
G \models \exists y_{1}, \ldots, \exists y_{n}\left(\psi\left(\bar{a} ; y_{1}, \ldots, y_{n}\right) \wedge \bigwedge_{1 \leq i \leq n} \phi_{i}\left(y_{i}\right)\right),
$$

we find $g_{1}, \ldots, g_{n}$ in $G$ such that

$$
G \models\left(\psi\left(\bar{b} ; g_{1}, \ldots, g_{n}\right) \wedge \bigwedge_{1 \leq i \leq n} \phi_{i}\left(g_{i}\right)\right),
$$

and thus we must have $g_{i}=d_{i}$ for all $i$. Therefore $\psi\left(\bar{x} ; d_{1}, \ldots, d_{n}\right) \in t p^{G}(\bar{b} \mid d c l(P))$. Thus $t p^{G}(\bar{a} \mid d c l(P)) \subseteq t p^{G}(\bar{b} \mid d c l(P))$ and by symmetry we conclude that $t p^{G}(\bar{a} \mid d c l(P))=t p^{G}(\bar{b} \mid d c l(P))$ as required.

(2) The proof is similar to that of (1) and is left to the reader.

Proof of Theorem 1.1. Let $F$ be a nonabelian group of finite rank. Let $\bar{a}, \bar{b} \in F^{n}$ and let $P \subseteq F$ such that $t p^{F}(\bar{a} \mid P)=t p^{F}(\bar{b} \mid P)$. 
By Lemma $6.3, t p^{F}(\bar{a} \mid d c l(P))=t p^{F}(\bar{b} \mid d c l(P))$, and by Theorem $6.2, \operatorname{dcl}(P)$ is finitely generated. Let $\bar{d}$ be a basis of $d \operatorname{cl}(P)$. We notice that $P \subseteq \operatorname{dcl}(P)$.

Then $t p^{F}(\bar{a}, \bar{d})=t p^{F}(\bar{b}, \bar{d})$ and thus there exists an automorphism $\sigma$ sending $\bar{a}$ to $\bar{b}$ and fixing $\bar{d}$ by Proposition 5.8. Thus in particular $\sigma$ fixes $P$.

Proof of Theorem 1.3. Similar to that of Theorem 1.1, the details are left to the reader.

\section{Two-Generated Torsion-Free Hyperbolic Groups}

In this section we give the proof of Theorem 1.5 and Corollary 1.7. We see that Corollary 1.7 is a mere consequence of Theorem 1.5 and Lemma 3.5. It remains to show Theorem 1.5. We first show the following lemma.

Lemma 7.1. Let $\Gamma$ be a torsion-free hyperbolic group and let $\Gamma_{0}$ be a subgroup of $\Gamma$. Suppose that $\Gamma=\left\langle H, t \mid U^{t}=V\right\rangle$, where $U$ and $V$ are cyclic malnormal subgroups of $H$. If $\Gamma_{0}$ is rigid then it is elliptic in the preceding splitting.

Proof. Suppose as a contradiction that $\Gamma_{0}$ is not elliptic. Since $\Gamma_{0}$ is freely indecomposable, $\Gamma_{0}$ admits a cyclic splitting $\Lambda$ inherited from the given splitting of $\Gamma$. This splitting is not essential because $\Gamma_{0}$ is rigid. It follows that the graph corresponding to $\Lambda$ is a tree, as otherwise $\Gamma_{0}$ can be written as an HNN-extension, contradicting again the rigidity of $\Gamma_{0}$. In particular, $\Gamma_{0}$ is an iterated amalgamated free product.

If each vertex group of $\Lambda$ is abelian, by the transivity of the commutation, $\Gamma_{0}$ itself is abelian; which is a contradiction.

Let $A_{0}$ be a nonabelian vertex group. We claim that each vertex group connected to $A_{0}$ is cylic. Let $V_{0}$ be the vertex corresponding to $A_{0}$ and let $V_{1}$ be another vertex connected to $V_{0}$ by $e$. Let $\Lambda^{\prime}$ be the graph obtained by deleting $e$ from $\Lambda$. Then $\Gamma_{0}=L_{1} *_{a=b} L_{2}$, where $L_{1}$ and $L_{2}$ are the fundamental groups of the connected components of $\Lambda^{\prime}$. Then $A_{0} \leq L_{1}$ or $A_{0} \leq L_{2}$ and without loss of generality we assume that $A_{0} \leq L_{1}$. Hence $L_{1}$ is nonabelian. If $L_{2}$ is nonabelian, then $\Gamma_{0}$ admits a principal cyclic splitting; a contradiction. Therefore, $L_{2}$ is abelian and thus cyclic. The vertex group corresponding to $V_{1}$ is contained in $L_{2}$ and thus cyclic as claimed.

Let $B_{0}$ be a vertex group corresponding to a vertex $V_{1}$ connected to $V_{0}$ by $e$. Since the splitting of $\Gamma_{0}$ is inherited from that of $\Gamma$, the fundamental group of the graph of groups consisting of $V_{0}, V_{1}$ and $e$ is of the form $L=A^{x} *_{a^{x}=b^{y}} B^{y}$ where $A, B \leq H$ and $A_{0}=A^{x}, B_{0}=B^{y}, x, y \in \Gamma$.

We are going to show that $L$ is elliptic; that is $L$ is contained in a conjugate of $H$. We have $a, b \in H$ and $a=\left(x y^{-1}\right) b\left(y x^{-1}\right)$ and $L^{x^{-1}}=A *_{a=b y x-1} B^{y x^{-1}}$. If $y x^{-1} \in H$ then $L$ is elliptic as claimed. So we suppose that $y x^{-1} \notin H$.

Observe that $U \cap V^{h}=1$ for any $h \in H$; as otherwise $\Gamma$ would contain $\mathbb{Z}^{2}$ which is a contradiction with its hyperbolicity. By Lemma 3.1, one of the following cases 
holds:

(1) $b=u^{p \gamma}, a=v^{p \delta}, y x^{-1}=\gamma^{-1} t \delta$, where $p \in \mathbb{Z}$ and $\gamma, \delta \in H$;

(2) $b=v^{p \gamma}, a=u^{p \delta}, y x^{-1}=\gamma^{-1} t^{-1} \delta$, where $p \in \mathbb{Z}$ and $\gamma, \delta \in H$;

where we have assumed that $U$ and $V$ are generated by $u$ and $v$, respectively. We treat only case (1), the other case being similar. Since $B$ is cyclic and $b=u^{p \gamma}$ and $U$ is malnormal, we get $B=\left\langle u^{q \gamma}\right\rangle$ for some $q \in \mathbb{Z}$. Therefore

$$
B=\delta^{-1} \cdot t^{-1} \cdot \gamma\left\langle\gamma^{-1} u^{q} \gamma\right\rangle \gamma^{-1} \cdot t \cdot \delta=\left\langle v^{q}\right\rangle^{\delta} \leq H
$$

and thus $L$ is elliptic as claimed.

Let $\Lambda^{\prime \prime}$ be the graph of groups obtained by collapsing $e$. Then $\Lambda^{\prime \prime}$ has fewer vertices than $\Lambda$. Proceeding by induction on the number of vertices, we conclude that $\Gamma_{0}$ is elliptic; a final contradiction.

We are now in a position to prove Theorem 1.5. Let $\Gamma_{1} \leq \Gamma_{2} \leq \cdots \leq \Gamma_{n}=\Gamma$ be a sequence given by Theorem 3.2, where $\Gamma_{1}$ is rigid. Since $\Gamma_{1}$ is rigid, by Lemma 3.3, there exists a finite subset $S \subseteq \Gamma_{1} \backslash\{1\}$ such that for any endomorphism $\varphi$ of $\Gamma$ if $1 \notin \varphi(S)$ then $\varphi$ is one-to-one in restriction to $\Gamma_{1}$.

Let $\varphi$ be an endomorphism of $\Gamma$ such that $1 \notin \varphi(S)$ and let us show that $\varphi$ is an automorphism.

We have $\varphi\left(\Gamma_{1}\right) \leq \Gamma$ and $\Gamma=\left\langle\Gamma_{n-1}, t \mid A^{t}=B\right\rangle$. Now $\varphi\left(\Gamma_{1}\right)$ is isomorphic to $\Gamma_{1}$ and thus rigid. By Lemma 7.1, $\varphi\left(\Gamma_{1}\right)$ is elliptic in the above splitting; that is $\varphi\left(\Gamma_{1}\right)$ is in a conjugate of $\Gamma_{n-1}$. Using a similar argument and proceeding by induction, $\varphi\left(\Gamma_{1}\right)$ is in a conjugate of $\Gamma_{1}$.

Let $g \in \Gamma$ such that $\varphi\left(\Gamma_{1}\right) \leq g \Gamma_{1} g^{-1}$ and let $\tau_{g}(x)=x^{g}$. Therefore we have $\tau_{g} \circ \varphi\left(\Gamma_{1}\right) \leq \Gamma_{1}$. Since $\Gamma_{1}$ is co-hopfian and $\tau_{g} \circ \varphi$ is one-to-one in restriction to $\Gamma_{1}$, we conclude that $\tau_{g} \circ \varphi\left(\Gamma_{1}\right)=\Gamma_{1}$.

Set $\phi=\tau_{g} \circ \varphi$. We show by induction on $i$ that the restriction of $\phi$ to $\Gamma_{i}$ is an automorphism of $\Gamma_{i}$. Write

$$
\Gamma_{i+1}=\left\langle\Gamma_{i}, t_{i} \mid A_{i}^{t_{i}}=B_{i}\right\rangle, \quad A_{i}=\left\langle a_{i}\right\rangle, \quad B_{i}=\left\langle b_{i}\right\rangle .
$$

We claim that $\phi\left(t_{1}\right) \in \Gamma_{2}$. If $n=2$, then clearly $\phi\left(t_{1}\right) \in \Gamma_{2}$. Hence we suppose that $n \geq 3$.

Let us first prove that $\phi\left(t_{1}\right) \in \Gamma_{n-1}$. Suppose as a contradiction that $\phi\left(t_{1}\right) \notin$ $\Gamma_{n-1}$. We have

$$
\phi\left(t_{1}\right)^{-1} \phi\left(a_{1}\right) \phi\left(t_{1}\right)=\phi\left(b_{1}\right) \quad \text { and } \quad \phi\left(a_{1}\right), \phi\left(b_{1}\right) \in \Gamma_{1} \leq \Gamma_{n-1} .
$$

Observe that $A_{i} \cap B_{i}^{h}=1$ for any $h \in \Gamma_{i}$; as otherwise $\Gamma_{i+1}$ would contain $\mathbb{Z}^{2}$ which is a contradiction with its hyperbolicity. According to Lemma 3.1, one of the following cases holds:

(1) $\phi\left(a_{1}\right)=\gamma^{-1} a_{n-1}^{p} \gamma, \phi\left(b_{1}\right)=\delta^{-1} b_{n-1}^{p} \delta, \phi\left(t_{1}\right)=\gamma^{-1} t_{n-1} \delta$, where $p \in \mathbb{Z}$ and $\gamma, \delta \in \Gamma_{n-1}$. 
(2) $\phi\left(a_{1}\right)=\gamma^{-1} b_{n-1}^{p} \gamma, \phi\left(b_{1}\right)=\delta^{-1} a_{n-1}^{p} \delta, \phi\left(t_{1}\right)=\gamma^{-1} t_{n-1}^{-1} \delta$, where $p \in \mathbb{Z}$ and $\gamma, \delta \in \Gamma_{n-1}$.

Let us treat case (1), case (2) can be treated similarly. We first show that $p= \pm 1$. We have $a_{n-1}^{\gamma} \in C_{\Gamma_{n-1}}\left(\phi\left(a_{1}\right)\right)$. According to [9, Theorem 3.2(i)], $C_{\Gamma_{n-1}}\left(\phi\left(a_{1}\right)\right)=$ $C_{\Gamma_{n-2}}\left(\phi\left(a_{1}\right)\right)$. Repeated application of $\left[9\right.$, Theorem 3.2(i)] gives $C_{\Gamma_{n-1}}\left(\phi\left(a_{1}\right)\right)=$ $C_{\Gamma_{1}}\left(\phi\left(a_{1}\right)\right)$. Therefore $a_{n-1}^{\gamma} \in C_{\Gamma_{1}}\left(\phi\left(a_{1}\right)\right)$. Since the restriction of $\phi$ to $\Gamma_{1}$ is an automorphism, we find $c \in \Gamma_{1}$ such that $\phi(c)=a_{n-1}^{\gamma}$ and $a_{1}=c^{p}$. Since $a_{1}$ is root-free, we conclude finally that $p= \pm 1$ as claimed.

We now rewrite $\Gamma$ as follows:

$$
\Gamma=\left\langle\Gamma_{n-1}, s \mid s^{-1} \phi\left(a_{1}\right) s=\phi\left(b_{1}\right)\right\rangle,
$$

where $s=\phi\left(t_{1}\right)$. We also have

$$
\Gamma_{n-1}=\left\langle\Gamma_{n-2}, t_{n-2} \mid A_{n-2}^{t_{n-2}}=B_{n-2}\right\rangle, \quad \phi\left(a_{1}\right), \phi\left(b_{1}\right) \in \Gamma_{n-2} .
$$

Hence $\Gamma$ admits a principal cyclic splitting with more than one edge; a contradiction with $\left[12\right.$, Theorem A]. Therefore $\phi\left(t_{1}\right) \in \Gamma_{n-1}$ as claimed.

Using a similar argument and proceeding by induction, we conclude that $\phi\left(t_{1}\right) \in$ $\Gamma_{2}$. In particular $\phi\left(\Gamma_{2}\right) \leq \Gamma_{2}$.

Clearly $\phi\left(t_{1}\right) \notin \Gamma_{1}$; otherwise $a_{1}$ and $b_{1}$ are conjugate in $\Gamma_{1}$ and thus $\Gamma_{2}$ contains $\mathbb{Z}^{2}$ contradicting its hyperbolicity. Hence, by Lemma 3.1, one of the following cases holds

(1) $\phi\left(a_{1}\right)=\gamma^{-1} a_{1}^{p} \gamma, \phi\left(b_{1}\right)=\delta^{-1} b_{1}^{p} \delta, \phi\left(t_{1}\right)=\gamma^{-1} t_{1} \delta$, where $p \in \mathbb{Z}$ and $\gamma, \delta \in \Gamma_{1}$.

(2) $\phi\left(a_{1}\right)=\gamma^{-1} b_{1}^{p} \gamma, \phi\left(b_{1}\right)=\delta^{-1} a_{1}^{p} \delta, \phi\left(t_{1}\right)=\gamma^{-1} t_{1}^{-1} \delta$, where $p \in \mathbb{Z}$ and $\gamma, \delta \in \Gamma_{1}$.

Let us treat case (1), case (2) being similar. Proceeding as above, we have $p= \pm 1$. Again as before, we rewrite $\Gamma_{2}$ as

$$
\Gamma_{2}=\left\langle\Gamma_{1}, s \mid s^{-1} \phi\left(a_{1}\right) s=\phi\left(b_{1}\right)\right\rangle,
$$

where $s=\phi\left(t_{1}\right)$. Hence, we get $\phi\left(\Gamma_{2}\right)=\Gamma_{2}$ and in particular the restriction of $\phi$ to $\Gamma_{2}$ is an automorphism of $\Gamma_{2}$.

Applying the same argument and proceeding by induction, we conclude that for every $i$ the restriction of $\phi$ to $\Gamma_{i}$ is an automorphism of $\Gamma_{i}$. In particular, $\phi$ is an automorphism of $\Gamma$ as well as $\varphi$.

\section{Remarks}

(1) In the proof of Theorem 1.1, we have used the definable closure. The following lemma, of independant interest avoids the use of the definable closure.

Lemma 8.1. Let $G$ be a finitely generated equationally noetherian group and let $P$ be a subset of $G$. Then there exists a finite subset $P_{0} \subseteq P$ such that for any endomorphism $f$ of $G$, if $f$ fixes $P_{0}$ pointwise then $f$ fixes $P$ pointwise. 
Proof. Let $\bar{g}$ be a generating tuple of $G$. Write $P=\left\{p_{i} \mid i \in \mathbb{N}\right\}$. Then for every $i \in \mathbb{N}$, there exists a word $w_{i}(\bar{x})$ such that $p_{i}=w_{i}(\bar{g})$. Since $G$ is equationally noetherian, there exists $n \in \mathbb{N}$ such that

$$
G \models \forall \bar{x}\left(\left(p_{0}=w_{0}(\bar{x}) \wedge \cdots \wedge p_{n}=w_{n}(\bar{x})\right) \Longrightarrow p_{i}=w_{i}(\bar{x})\right),
$$

for any $i \in \mathbb{N}$.

Let $P_{0}=\left\{p_{0}, \ldots, p_{n}\right\}$ and $f$ an endomorphism such that $f\left(p_{i}\right)=p_{i}$ for every $0 \leq i \leq n$. Therefore $p_{i}=f\left(p_{i}\right)=w_{i}(f(\bar{g}))$ for any $0 \leq i \leq n$. Hence, by (8.1), $p_{i}=w_{i}(f(\bar{g}))$ for any $i \in \mathbb{N}$ and thus $p_{i}=f\left(p_{i}\right)$ for any $i \in \mathbb{N}$ as required.

Now let $\bar{a}$ and $\bar{b}$ be tuples from $F$ such that $\operatorname{tp}^{F}(\bar{a} \mid P)=t p^{F}(\bar{b} \mid P)$. Then $t p^{F}\left(\bar{a} \mid P_{0}\right)=t p^{F}\left(\bar{b} \mid P_{0}\right)$ and $t p^{F}\left(\bar{a}, P_{0}\right)=t p^{F}\left(\bar{b}, P_{0}\right)$. By Proposition 5.8, there exists an automorphism $f$ such that $f(\bar{a})=\bar{b}$ and which fixes $P_{0}$ pointwise. Hence $f$ fixes $P$ pointwise.

(2) We note that a non-free two-generated torsion-free hyperbolic group is not necessarily rigid. Here is an example. Let $F=\langle a, b \mid\rangle$ be the free group of rank 2 and let $r \in F$ satisfying the following properties:

(i) $r$ is root-free, is cyclically reduced and its length is greater than 6;

(ii) the symmetrized set generated by $r$ satisfies $C^{\prime}(1 / 8)$.

Let $\Gamma=\langle a, b \mid r=1\rangle$. By [13, Theorem 5.4, V], $a$ and $b$ are not conjugate in $\Gamma$. It follows in particular that no power of $a$ is conjugate to a power of $b$. We also see that $\langle a \mid\rangle$ and $\langle b \mid\rangle$ are malnormal in $\Gamma$. Hence the HNN-extension $L=\left\langle\Gamma, t \mid a^{t}=b\right\rangle$ is conjugately separated in the sense of [10]. Since $\Gamma$ is torsion-free and hyperbolic, by [10, Corollary 1], $L$ is a torsion-free hyperbolic group. Hence $L$ is a non-free twogenerated torsion-free hyperbolic group which admits an essential cyclic splitting; and thus $L$ is not rigid.

(3) We give the proof that if $\Gamma$ is a two-generated non-free torsion-free hyperbolic group, then $\Gamma$ is an elementary subgroup of $\Gamma * \mathbb{Z}$, using results from [39]. Let $\Gamma$ be a torsion-free hyperbolic group. In [39], Sela shows that if $\Gamma$ is not elementary equivalent to a free group, then $\Gamma$ has a smallest elementary subgroup, denoted by $E C(\Gamma)$, called the elementary core of $\Gamma$.

For the definition of JSJ-decompositions for torsion-free hyperbolic groups and theirs properties, we refer the reader to [34], and for a more general treatment $[5,6]$. The following notion is used implicitly in [39].

Definition 8.2. ([29, Definition 5.9]) Let $\Gamma$ be a torsion-free hyperbolic group, let $A$ be a subgroup of $\Gamma$ and let $\Lambda$ be the cyclic JSJ-decomposition of $A$. A homomorphism from $A$ to $\Gamma$ is a preretraction if its restriction to each non-surface type vertex group $A_{v}$ of $\Lambda$ is just a conjugation by some element $g_{v}$ of $\Gamma$, and if surface type vertex groups have a nonabelian images. 
The following proposition is used implicitly in [39].

Proposition 8.3. ([29, Proposition 5.12]) Let $\Gamma$ be a torsion-free hyperbolic group. Let $A$ be a noncyclic retract of $\Gamma$ which admits a cyclic JSJ-decomposition $\Lambda$. Suppose $\Gamma^{\prime}$ is a subgroup of $\Gamma$ containing $A$ such that either $\Gamma^{\prime}$ is a free factor of $\Gamma$, or $\Gamma^{\prime}$ is a retract of $\Gamma$ by a retraction $r: \Gamma \rightarrow \Gamma^{\prime}$ which makes $\left(\Gamma, \Gamma^{\prime}, r\right)$ a hyperbolic floor. If there exists a non-injective preretraction $A \rightarrow \Gamma$ with respect to $\Lambda$, then there exists a non-injective preretraction $A \rightarrow \Gamma^{\prime}$ with respect to $\Lambda$.

Definition 8.4. Let $\Gamma$ be a noncyclic torsion-free hyperbolic group. We say that $\Gamma$ is hyperbolically minimal if every preretraction from $\Gamma$ to $\Gamma$ is an automorphism.

Definition 8.5. ([39, Definition 7.3]) Let $\Gamma$ be a noncyclic torsion-free hyperbolic group. $\Gamma$ is said to be an elementary-prototype if:

(i) $\Gamma$ is not an $\omega$-residually free tower (i.e. $\Gamma$ is not elementary equivalent to a free group).

(ii) $\Gamma$ admits a Grushko free decomposition $\Gamma=H_{1} * \cdots * H_{p}$, where $H_{1}, \ldots, H_{p}$ are freely indecomposable, noncyclic and are not elementary free towers.

(iii) Each of the factors $H_{i}$ is hyperbolically minimal.

Definition 8.6. ([39, Definition 7.5]) Let $\Gamma$ be a noncyclic torsion-free hyperbolic group. The elementary core of $\Gamma$, denoted $E C(\Gamma)$, is defined iteratively as follows:

Let $\Gamma=H_{1} * \cdots * H_{m} * F$ be the Grushko decomposition of $\Gamma$, where each of the factors $H_{i}$ is noncyclic and freely indecomposable, and $F$ is a (possibly trivial) free group. Up to reordering, we let $H_{1}, \ldots, H_{t}$ be the factors that are not isomorphic to a closed surface group that is elementary equivalent to a free group, i.e. a hyperbolic surface group, where the surface is not the non-orientable surface of genus 2. If there are no such factors, we define $E C(\Gamma)=1$. Otherwise, we define $E C(\Gamma)=E C\left(H_{1}\right) * \cdots * E C\left(H_{t}\right)$.

To construct the elementary core of a freely indecomposable, noncyclic factor $H_{i}$, we associate with it its cyclic JSJ decomposition.

(i) If $H_{i}$ is hyperbolically minimal, we set $E C\left(H_{i}\right)=H_{i}$, and conclude the construction of the elementary core of the factor $H_{i}$.

(ii) If $H_{i}$ is not hyperbolically minimal, there must exist a non-injective preretraction $r_{0}: H_{i} \rightarrow H_{i}$. Proposition 8.3 implies the existence of a proper retraction $r: H_{i} \rightarrow H_{i}$ which makes $H_{i}$ a hyperbolic floor. Continuing the construction iteratively, we set the elementary core of the factor $H_{i}$ to be $E C\left(H_{i}\right)=E C(r(H i))$.

In each step along the iterative construction of the elementary core of the torsion-free hyperbolic group $\Gamma$, we replace a factor by a proper retract of it, which is a proper quotient of the factor, and a subgroup of the original torsionfree hyperbolic group $\Gamma$. Hence, the descending chain condition for $\Gamma$-limit groups 
proves that the construction of the elementary core terminates after finitely many steps.

Proposition 8.7. If $\Gamma$ is a non-free two-generated torsion-free hyperbolic group, then $E C(\Gamma)=\Gamma$. In particular, $\Gamma$ is an elementary subgroup of $\Gamma * \mathbb{Z}$.

Proof. If $\Gamma$ is rigid then the cyclic JSJ-decomposition of $\Gamma$ is trivial and in particular $\Gamma$ is hyperbolically minimal. Therefore $E C(\Gamma)=\Gamma$.

So suppose that $\Gamma$ admits a principal cyclic splitting. By $[12$, Theorem A], the cyclic JSJ-decomposition of $\Gamma$ has one vertex and one edge. We claim that $\Gamma$ is hyperbolically minimal. Write

$$
\Gamma=\left\langle H, t \mid U^{t}=V\right\rangle,
$$

the cyclic splitting corresponding to the cyclic JSJ-decomposition of $\Gamma$. Clearly $H$ is not a surface type vertex group. Hence, we must show that if $f$ is an endomorphism of $\Gamma$ whose restriction to $H$ is a conjugation by an element $g$, then $f$ is an automorphism.

Let $\tau_{g}(x)=x^{g^{-1}}$ be the conjugation by $g^{-1}$. Then the restriction of $\tau=\tau_{g} \circ f$ to $H$ is the identity on $H$. It is sufficient to show that $\tau(t)=h_{0} t h_{1}$ for some $h_{0}, h_{1} \in H$. The proof uses normal forms as in Lemma 3.1. Since $\Gamma$ is a CSA-group, either $U$ or $V$ is malnormal in $H$ and without loss of generality we assume that $U$ is malnormal.

Let $s=\tau(t)$ and write $s=h_{0} t^{\epsilon_{0}} \cdots t^{\epsilon_{n}} h_{n+1}$ in normal form. Hence

$$
h_{n+1}^{-1} t^{-\epsilon_{n}} \cdots t^{-\epsilon_{0}} h_{0}^{-1} u h_{0} t^{\epsilon_{0}} \cdots t^{\epsilon_{n}} h_{n+1}=v,
$$

and thus either $h_{0}^{-1} u h_{0} \in U$ and $\epsilon_{0}=1$ or $h_{0}^{-1} u h_{0} \in V$ and $\epsilon_{0}=-1$. Since $U^{h} \cap V=1$ for any $h \in H$, the second case is impossible. Therefore $h_{0} \in U$ as $U$ is malnormal.

We claim that $n=0$. Suppose first that $n \geq 2$. Then $h_{1}^{-1} v h_{1} \in U$ and $\epsilon_{1}=1$ or $h_{1}^{-1} v h_{1} \in V$ and $\epsilon_{1}=-1$. Since $U^{h} \cap V=1$, the first case is impossible. Therefore we have the second case and thus $h_{1} \in C_{H}(V)$ and $h_{1}^{-1} v h_{1}=v$. Using similar argument, we conclude that $h_{2}^{-1} u h_{2} \in U$ and $\epsilon_{2}=1$. Hence $h_{2} \in U$ and the sequence $\left(t^{\epsilon_{1}}, h_{2}, t^{\epsilon_{2}}\right)$ is not reduced, a contradiction.

We conclude that $n \leq 1$. If we assume that $n=1$, we get that $u$ and $v$ are conjugate by an element from $H$; a contradiction. Therefore $n=0$ as required. Hence $\tau$ is an automorphism as well as $f$.

Since $\Gamma$ is freely indecomposable, we conclude using the definition of the elementary core that $E C(\Gamma)=\Gamma$.

By definition of the elementary core, we have $E C(\Gamma * \mathbb{Z})=E C(\Gamma)$ and thus $\Gamma$ is an elementary subgroup of $\Gamma * \mathbb{Z}$ by [39, Theorem 7.6].

(4) It is noted in [30] that a nonabelian free group is connected. Hence, one may ask if this is still true for nonabelian torsion-free hyperbolic groups. Recall that 
a group $G$ is said to be connected, if $G$ is without a definable subgroup of finite index.

Proposition 8.8. A noncyclic torsion-free hyperbolic group is connected.

Recall that a definable subset $X$ of $G$ is said to be right generic, if there exist $g_{1}, \ldots, g_{n} \in G$ such that $G=g_{1} X \cup \cdots \cup g_{n} X$. Left generic definable subsets are defined analogously. Now we show the following lemma.

Lemma 8.9. Let $G$ be a group and suppose that $G$ satisfies the following: if $X$ and $Y$ are right generic sets then $X \cap Y \neq \emptyset$. Then $G$ is connected.

Proof. If $H$ is a definable subgroup of finite index, then $G=g_{1} H \cup \cdots \cup g_{n} H$. Then any $g_{i} H$ is right generic because

$$
G=\left(g_{1} g_{i}^{-1}\right) g_{i} H \cup \cdots\left(g_{n} g_{i}^{-1}\right) g_{i} H,
$$

and therefore for any $i, j$ we have $g_{i} H \cap g_{j} H \neq \emptyset$ and thus we must have $G=H$.

For a group $G$, we denote by $G[a]$ the group $G * \mathbb{Z}$ where $a$ is a generating element of $\mathbb{Z}$. The following lemma is a slight refinement of an observation by Poizat.

Lemma 8.10. Let $G$ be a group and suppose that $G \preceq G[a]$. If $\phi(G)$ is a right generic subset of $G$, then $a \in \phi(G[a])$. In particular $G$ is connected.

Proof. Suppose that $G=g_{1} \phi(G) \cup \cdots \cup g_{n} \phi(G)$. Since $G \preceq G[a]$, we also have $G[a]=g_{1} \phi(G[a]) \cup \cdots \cup g_{n} \phi(G[a])$. Therefore for some $i, g_{i}^{-1} a \in \phi(G[a])$. Since there exists an automorphism of $G[a]$ which sends $g_{i}^{-1} a$ to $a$, we get $a \in \phi(G[a])$.

Since $G \preceq G[a]$, it follows that if $X$ and $Y$ are right generic subsets, then $X \cap Y \neq \emptyset$. Therefore by Lemma 8.9, $G$ is connected.

Let $\Gamma$ be a torsion-free hyperbolic group. We need the following properties of the elementary core $E C(\Gamma)$, which follow from [39].

Fact 8.11. The elementary core satisfies the following properties:

(1) $E C(\Gamma)=1$ if and only if $\Gamma$ is elementary equivalent to a nonabelian free group.

(2) $E C(\Gamma)=E C(\Gamma * \mathbb{Z})$ and $E C(E C(\Gamma))=E C(\Gamma)$.

(3) If $E C(\Gamma) \neq 1$ then $E C(\Gamma) \preceq \Gamma$.

By $(2)$ and (3) we get $E C(\Gamma) \preceq E C(\Gamma) * \mathbb{Z}$. We conclude, by Lemma 8.10, that $E C(\Gamma)$ is connected and thus by elementary equivalence, $\Gamma$ is connected.

\section{Acknowledgment}

The author wishes to express his thanks to A. Ivanov and G. Sabbagh for suggesting several questions and to C. Lasserre for some remarks. 


\section{References}

1. I. Belegradek, Aspherical manifolds with relatively hyperbolic fundamental groups, Geom. Dedicata 129 (2007) 119-144.

2. G. Baumslag, A. Myasnikov and V. Remeslennikov, Algebraic geometry over groups. I. Algebraic sets and ideal theory, J. Algebra 219 (1999) 16-79.

3. C. Champetier and V. Guirardel, Limit groups as limits of free groups, Israel J. Math. 146 (2005) 1-75.

4. T. Delzant, Sous-groupes à deux générateurs des groupes hyperboliques, in Group Theory from a Geometrical Viewpoint (Trieste, 1990) (World Scientific, 1991), pp. $177-189$.

5. V. Guirardel and G. Levitt, JSJ decompositions: Definitions, existence, uniqueness. I: The JSJ deformation space, preprint.

6. V. Guirardel and G. Levitt, JSJ decompositions: Definitions, existence, uniqueness. II. Compatibility and acylindricity, preprint.

7. D. Groves and H. Wilton, Conjugacy classes of solutions to equations and inequations over hyperbolic groups, 2007.

8. W. Hodges, Model Theory, Encyclopedia of Mathematics and its Applications, Vol. 42 (Cambridge Univ. Press, 1993).

9. E. Jaligot and A. Ould Houcine, Existentially closed CSA-groups, J. Algebra 280 (2004) $772-796$.

10. O. Kharlampovich and A. Myasnikov, Hyperbolic groups and free constructions, Trans. Amer. Math. Soc. 350 (1998) 571-613.

11. O. Kharlampovich and A. Myasnikov, Elementary theory of free non-abelian groups, J. Algebra 302 (2006) 451-552.

12. I. Kapovich and R. Weidmann, On the structure of two-generated hyperbolic groups, Math. Z. 231 (1999) 783-801.

13. R. C. Lyndon and P. E. Schupp, Combinatorial Group Theory (Springer-Verlag, 1977).

14. D. Marker, Model Theory: An Introduction (Springer-Verlag, 2002).

15. A. Nies, Aspects of free groups, J. Algebra 263 (2003) 119-125.

16. A. Nies, Separating classes of groups by first-order sentences, Internat. J. Algebra Comput. 13 (2003) 287-302.

17. A. Nies, Comparing quasi-finitely axiomatizable and prime groups, J. Group Theory 10 (2007) 347-361.

18. A. Ould Houcine, Limit groups of equationally noetherian groups, in Geometric Group Theory (Birkhäuser, 2007), pp. 103-119.

19. A. Ould Houcine, On superstable CSA-groups, Ann. Pure Appl. Logic 154 (2008) $1-7$.

20. A. Ould Houcine, On finitely generated models of theories with at most countably many nonisomorphic finitely generated models, submitted.

21. A. Ould Houcine, Note on the Cantor-Bendixson rank of limit groups, Commun. Algebra 38 (2010) 2825-2831.

22. A. Ould Houcine, Subgroup theorem for valuated groups and the csa property, $J$. Algebra 134 (2010) 159-182.

23. A. Ould Houcine and D. Vallino, Algebraic and definable closure in free groups, in preparation.

24. A. Yu. Ol'shanskiı̌, An infinite group with subgroups of prime orders, Izv. Akad. Nauk SSSR Ser. Mat. 44 (1980) 309-321.

25. A. Yu. Ol'shanskiı̌, Geometry of Defining Relations in Groups, Mathematics and its Applications (Soviet Series), Vol. 70 (Kluwer Academic Press, 1991), translated from the 1989 Russian original by Yu. A. Bakhturin. 
26. F. Oger and G. Sabbagh, Quasi-finitely axiomatizable nilpotent groups, J. Group Theory 9 (2006) 95-106.

27. J. C. O'Neill and E. C. Turner, Test elements and the retract theorem in hyperbolic groups, New York J. Math. 6 (2000) 107-117.

28. C. Perin, Plongements élémentaires dans un groupe hyperbolique sans torsion, Thèse de doctorat, Université de Caen/Basse-Normandie, 2008.

29. C. Perin, Elementary embeddings in torsion-free hyperbolic groups, arXiv:0903.0945.

30. A. Pillay, Forking in the free group, J. Inst. Math. Jussieu 7 (2008) 375-389.

31. A. Pillay, On genericity and weight in the free group, Proc. Amer. Math. Soc. 137 (2009) 3911-3917.

32. C. Perin and R. Sklinos, Homogeneity in the free group, preprint.

33. E. Rips and Z. Sela, Structure and rigidity in hyperbolic groups. I, Geom. Funct. Anal. 4 (1994) 337-371.

34. E. Rips and Z. Sela, Cyclic splittings of finitely presented groups and the canonical JSJ decomposition, Ann. Math. (2) 146 (1997) 53-109.

35. Z. Sela, Structure and rigidity in (Gromov) hyperbolic groups and discrete groups in rank 1 Lie groups. II, Geom. Funct. Anal. 7 (1997) 561-593.

36. Z. Sela, Diophantine geometry over groups. $\mathrm{V}_{1}$. Quantifier elimination. I, Israel J. Math. 150 (2005) 1-197.

37. Z. Sela, Diophantine geometry over groups. $\mathrm{V}_{2}$. Quantifier elimination. II, Geom. Funct. Anal. 16 (2006) 537-706.

38. Z. Sela, Diophantine geometry over groups. VI. The elementary theory of a free group, Geom. Funct. Anal. 16 (2006) 707-730.

39. Z. Sela, Diophantine geometry over groups. VII. The elementary theory of a hyperbolic group, Proc. Lond. Math. Soc. 99 (2009) 217-273. 